\title{
Radiation from laser accelerated electron bunches: coherent terahertz and femtosecond x-rays
}

\author{
W. P. Leemans, Member, IEEE, E. Esarey, J. van Tilborg, P. A. Michel, C. B. Schroeder, Cs. Tóth, \\ C. G. R. Geddes, and B. A. Shadwick
}

\begin{abstract}
Electron beam based radiation sources provide electromagnetic radiation for countless applications. The properties of the radiation are primarily determined by the properties of the electron beam. Compact laser driven accelerators are being developed that can provide ultra-short electron bunches (femtosecond duration) with relativistic energies reaching towards a GeV. The electron bunches are produced when an intense laser interacts with a dense plasma and excites a large amplitude plasma density modulation (wakefield) that can trap background electrons and accelerate them to high energies. The short pulse nature of the accelerated bunches and high particle energy offer the possibility of generating radiation from one compact source that ranges from coherent terahertz to gamma rays. The intrinsic synchronization to a laser pulse and unique character of the radiation offers a wide range of possibilities for scientific applications. Two particular radiation source regimes are discussed: coherent terahertz emission and x-ray emission based on betatron oscillations and Thomson scattering.
\end{abstract}

Index Terms - Lasers, Plasmas, Electron accelerators, Electromagnetic radiation, $\mathrm{X}$-rays

\section{INTRODUCTION}

$\mathbf{L}$ ASER-driven, plasma-based accelerators [1] hold the potential of becoming compact alternatives to conventional radio-frequency-based linear accelerators (linacs). Accelerating gradients in the 10's to 100 's of $\mathrm{GV} / \mathrm{m}$ have been measured in laser wakefield accelerator (LWFA) experiments [2]-[8] $\mathrm{m}$ which are three orders of magnitude higher than in conventional linacs. In the self-modulated LWFA regime, acceleration of electrons has also been demonstrated, [2][8] with bunches containing up to several $\mathrm{nC}$ of charge and energy spectra characterized by a Boltzmann-like distribution

Manuscript received August 4, 2004; revised August 18, 2004. This work was supported by the United States Department of Energy.

W. P. Leemans is with the Lawrence Berkeley National Laboratory, University of California, Berkeley, CA 94720.

E. Esarey is with the Lawrence Berkeley National Laboratory, University of California, Berkeley, CA 94720.

J. van Tilborg is with the Lawrence Berkeley National Laboratory, University of California, Berkeley, CA 94720 and also with the Technische Universiteit Eindhoven, the Netherlands.

P. A. Michel is with the Lawrence Berkeley National Laboratory, University of California, Berkeley, CA 94720.

C. B. Schroeder is with the Lawrence Berkeley National Laboratory, University of California, Berkeley, CA 94720.

Cs. Tóth is with the Lawrence Berkeley National Laboratory, University of California, Berkeley, CA 94720.

C. G. R. Geddes is with the Lawrence Berkeley National Laboratory, University of California, Berkeley, CA 94720 and also with the University of California, Berkeley, USA.

B A. Shadwick is with the Lawrence Berkeley National Laboratory, University of California, Berkeley, CA 94720 and also the Institute for Advanced Physics, Conifer, CO 80433. with an equivalent temperature ranging from the few $\mathrm{MeV}$ to tens of $\mathrm{MeV}$. In this regime the laser pulse is modified during its propagation in the plasma leading to large amplitude plasma waves capable of trapping and accelerating background plasma electrons. Work is also under way to produce narrow energy spread beams using laser triggered injection techniques [9]-[14] and via control of the plasma properties (transverse and longitudinal) and laser parameters. Recent experiments at Lawrence Berkeley National Laboratory (LBNL) have demonstrated the production of narrow energy spread (few percent), high energy $(\sim 100 \mathrm{MeV})$, high charge $(\sim 0.5 \mathrm{nC})$ electron bunches from a LWFA that used a preformed plasma density channel [15]. Narrow energy electron bunches were also observed in the UK and France [16], [17].

Although the use of laser-plasma accelerators for high energy physics applications will require performance well beyond todays achievements, several applications with less stringent beam property requirements are becoming possible. One of these is radio-isotope production through $(\gamma, \mathrm{n})$ reactions with laser accelerated bunches [18], [19], which only requires a sufficient number of electrons with energy in excess of 10 's of MeV, a condition that is relatively straightforward to obtain using LWFAs. Two other applications are being explored which exploit the short pulse nature and high charge of the accelerated bunches. The first is the production of coherent terahertz $\left(10^{12} \mathrm{~Hz}\right)$ radiation when the femtosecond electron bunches cross the plasma-vacuum boundary and emit transition radiation [20]-[22]. The second aims at the generation of femtosecond $\mathrm{x}$-ray pulses, which are produced either by the betatron radiation emitted as the electron beam propagates through the plasma, or via Thomson scattering of an intense laser off the electron beam. These latter applications are the focus of the present paper.

The first part of this paper provides an overview of recent experiments [20], [22] and theory [21] on generation of coherent terahertz radiation. This electron beam-based radiation source relies on two novel components: (1) extremely dense, sub-ps electron bunches produced with a compact laser-plasma accelerator and (2) the production of coherent transition radiation by these bunches at the boundary between a plasma and vacuum. This source has the potential for generating up to tens of $\mu \mathrm{J}$ per pulse, several orders of magnitude beyond conventional THz radiation sources. The theory of transition radiation generated by a relativistic electron bunch crossing a plasmavacuum boundary is summarized in Sec. II-A. Sections II-B II-F discuss the experimental set-up and present evidence for the coherent nature of the radiation at a frequency of $94 \mathrm{GHz}$ 
and in the 0.3 to $19 \mathrm{THz}$ range, polarization measurements, and preliminary frequency spectrum measurements. Using these preliminary spectral measurements, bunch durations on the order of 30-50 fs are inferred. Also compared are the measured and calculated total radiated energy. Methods for increasing the radiated energy beyond what has been achieved in the present experiments are discussed in the conclusion.

The second part of the paper discusses two methods for generating femtosecond $\mathrm{x}$-ray pulses. The first method relies on the emission of betatron radiation, i.e., synchrotron radiation emitted as the accelerated electrons undergo betatron oscillations within a plasma channel [23], [24]. These betatron oscillations are due to the radial electric field component of the large amplitude plasma wave (wake) excited within a plasma channel, the axial component of which is responsible for electron acceleration. This process can occur in both laserdriven and electron beam-driven plasma based accelerators. Betatron radiation from a plasma driven by an intense electron or laser beam has recently been observed [25], [26]. Section III-A presents the theory describing the betatron radiation mechanism, various scaling laws, and examples for the design of such an x-ray source.

Alternatively, $x$-ray radiation may be produced via Thomson scattering (TS) using an external laser pulse [27]-[33]. The first demonstration of the generation of sub-picosecond duration x-ray pulses using TS was implemented at the Beam Test Facility of the Advanced Light Source at LBNL [28][30]. The scattering geometry was configured for $90^{\circ}$ [34] so that the generated x-ray pulse duration of 300 fs (FWHM) was determined by the convolution between the laser pulse duration (100 fs) and the crossing time of the laser across the tightly focused electron beam (200-250 fs). The number of $\mathrm{X}$-rays and peak brightness of the $90^{\circ} \mathrm{TS}$ source experiments at LBNL were in part limited by the fact that the laser beam only interacted with about a $100 \mathrm{fs}$ long electron beam slice (or $0.3 \%$ of all the available electrons), as well as by the relatively high transverse emittance of the electron beam and low peak laser power used in the experiment. To increase the photon yield and source brightness, high quality femtosecond electron bunches are needed to enable femtosecond x-ray production through $180^{\circ}$ laser backscattering [35]. As indicated above, laser driven accelerators are now capable of producing such intense femtosecond electron bunches. Section III-B presents the theory describing the Thomson scattered radiation spectrum, and presents examples of TS x-ray sources for various electron energy distributions produced by laser-plasma accelerators.

\section{COHERENT TERAHERTZ RADIATION FROM LASER ACCELERATED BUNCHES}

Laser-triggered solid-state based sources of $\mathrm{THz}$ radiation have been developed that rely on switched photoconducting antennas (e.g., Ref. [36] and references therein) or optical rectification of femtosecond pulse trains [37]. Large aperture biased GaAs structures, operated at $1 \mathrm{kHz}$ repetition rate, have produced on the order of $0.5 \mu \mathrm{J} /$ pulse [38]. Most other sources using either laser switched structures or optical rectification have operated at high frequency (10's of MHz) with $\mu \mathrm{W}-\mathrm{mW}$ level average power. To date, the experiments carried out at the L'OASIS facility of LBNL [20] have collected and focused coherent radiation with energies near the $100 \mathrm{~nJ} /$ pulse level, in which the measured energy was limited primarily by the small collection angle $(\sim 100 \mathrm{mrad})$ and the small transverse size $(\sim 100 \mu \mathrm{m})$ of the plasma.

\section{A. Transition Radiation Theory}

Modeling of the radiation process can be done by assuming that the plasma (with dielectric constant $\epsilon=1-\omega_{p}^{2} / \omega^{2}$, where $\omega_{p}$ and $\omega$ are the plasma and radiation frequencies, respectively) is equivalent to a conductor with a sharp conductorvacuum boundary. The transition radiation will be generated by electron beam induced polarization currents at plasma densities below the critical density (where $\omega_{p}=\omega$ ) for the radiation wavelength. The plasma density profile used in the experiments discussed in Sec. II-B had a sufficiently large gradient such that the dielectric constant satisfied $|\epsilon| \gg 1$ within a distance on the order of a skin depth, and therefore the plasma can be well-modeled as a conductor for frequencies $\omega<\omega_{p}$. In addition, provided the plasma scale length is short compared to the radiation formation length, then the dielectric interface radiates as if it were a sharp dielectricvacuum boundary.

The energy radiated, per unit frequency $d \omega$ and per unit solid angle $d \Omega$, from a single electron traversing the dielectric boundary is given by the well-known result [39]-[41]

$$
\frac{d^{2} W_{e}}{d \omega d \Omega}=\left(\frac{r_{e} m_{e} c}{\pi^{2}}\right) \frac{u^{2}\left(1+u^{2}\right) \sin ^{2} \theta}{\left(1+u^{2} \sin ^{2} \theta\right)^{2}},
$$

where $\theta$ is the observation angle with respect to the electron trajectory (assumed to be normal to the plasma surface and along the $z$-axis), $u=\gamma v / c$ is the electron momentum normalized to $m_{e} c, c$ is the speed of light, $m_{e}$ is the electron rest mass, and $r_{e}=e^{2} / m_{e} c^{2}$ is the classical electron radius. The radiation pattern is zero along the axis $(\theta=0)$ and peaks at a radiation cone angle of $\theta \sim 1 / \gamma$ (assuming $\gamma \gg 1$ ). The differential energy radiated by a single electron $d^{2} W_{e} / d \omega d \Omega$ is independent of frequency. In practice, however, the maximum wavelength radiated will be limited, for example, by the physical dimensions of the system, as will be discussed below.

Integrating over solid angle $d \Omega=2 \pi \sin \theta d \theta$ yields

$$
\frac{d W_{e}}{d \omega}=\frac{2 r_{e} m_{e} c}{\pi}\left[\frac{\left(1+2 u^{2}\right)}{u \sqrt{1+u^{2}}} \tanh ^{-1}\left(\frac{u}{\sqrt{1+u^{2}}}\right)-1\right] \text {, }
$$

which, in the highly-relativistic limit $\gamma \gg 1$, reduces to $d W_{e} / d \omega \simeq(2 / \pi) r_{e} m_{e} c \ln (\gamma)$. The radiation from the beam of electrons sums incoherently for wavelengths short compared to the bunch length, i.e., $W_{\text {ITR }} \simeq N_{b} W_{e}$, where $N_{b}$ is the number of electrons in the bunch, and a monoenergetic divergenceless beam was assumed. For wavelengths long compared to the bunch length, the radiation sums coherently, i.e., $W_{\mathrm{CTR}} \simeq N_{b}^{2} W_{e}$. In particular, the total coherent radiated energy over all angles and frequencies is given by $W_{\text {tot }} \simeq$ $\left(4 r_{e} m_{e} c^{2}\right) N_{b}^{2} \ln (\gamma) / \lambda_{\min }$, assuming $\gamma \gg 1$, where $\lambda_{\min }$ is the minimum wavelength for which the bunch radiates coherently and is determined by the electron bunch dimensions, 
as will be discussed below. The total coherent energy can be written in practical units as

$$
W_{\text {tot }}[\mathrm{J}] \simeq 3.6 \times 10^{-2}(Q[\mathrm{nC}])^{2} \ln (\gamma) / \lambda_{\min }[\mu \mathrm{m}],
$$

where $Q$ is the bunch charge. For example, $\mathrm{Q}=5 \mathrm{nC}$ $\left(N_{b}=3.1 \times 10^{10}\right), \gamma=10$, and $\lambda_{\min }=200 \mu \mathrm{m}$ give $W_{\text {tot }} \simeq 10 \mathrm{~mJ}$, which is several orders of magnitude beyond that of conventional sources.

The above analysis can be generalized for the case of an arbitrary electron beam momentum distribution including the effects of coherence and the transverse size of the dielectric structure (diffraction from the plasma edge). Assuming a normalized energy distribution of the electron beam $g(u)$, the differential radiated energy by a bunch travelling normal to the plasma-vacuum boundary is [21]

$$
\begin{aligned}
& \frac{d^{2} W}{d \omega d \Omega}=\frac{r_{e} m_{e} c}{\pi^{2}} N_{b}\left(N_{b}-1\right) \sin ^{2} \theta \\
\times & \left|\int d u g(u) F(\omega, \theta, u) \frac{u\left(1+u^{2}\right)^{1 / 2}}{\left(1+u^{2} \sin ^{2} \theta\right)} D(\omega, \rho, \theta, u)\right|^{2},
\end{aligned}
$$

where

$$
F=\int d z d^{2} \vec{r}_{\perp} e^{-i \vec{k}_{\perp} \cdot \vec{r}_{\perp}} e^{-i \omega z / v} f\left(\vec{r}_{\perp}, z\right)
$$

is the Fourier transform of the electron beam spatial distribution $f\left(\vec{r}_{\perp}, z\right)$, or spatial form factor, $\vec{k}$ is the radiation wave vector, and

$$
\begin{aligned}
D=1-J_{0}(b u \sin \theta)\left[b K_{1}(b)\right. & \left.+b^{2} K_{0}(b) / 2\right] \\
& -b^{2} K_{0}(b) J_{2}(b u \sin \theta) / 2
\end{aligned}
$$

is the correction due to diffraction from the transverse edge of the plasma. Note that (5) assumes that there are no correlations between position and momentum. The parameter $b \equiv k \rho / u$ describes the relative influence of the transverse boundary (i.e., the ratio of the transverse size of the plasma $\rho$ to the transverse extent of the self-fields of the relativistic electrons $\sim \gamma \lambda$ ), and $K_{0}, K_{1}, J_{0}$, and $J_{2}$ are Bessel functions. For large transverse size (i.e., $b \gg 1$ ), $D \simeq 1$, and the coherent radiation is welldescribed by transition radiation. For $b \sim 1$, (i.e., transverse plasma size small compared to the effective source size $\gamma \lambda$ ) diffraction effects will strongly modify the radiated energy spectrum.

The degree of coherence in the bunch will be determined by the form factor (5). For a bunch with an uncorrelated Gaussian spatial distribution, the spatial form factor is $F=F_{\perp} F_{\|}$, where $F_{\perp}=\exp \left[-\left(k \sigma_{\perp} \sin \theta\right)^{2} / 2\right]$ and $F_{\|}=$ $\exp \left[-\left(\omega \sigma_{\|} / v\right)^{2} / 2\right]$, with $\sigma_{\perp}$ the rms transverse bunch radius and $\sigma_{\|}$the rms longitudinal bunch length. Fully-coherent emission in both the transverse and longitudinal directions requires $F \simeq 1$. This condition is satisfied when $\sigma_{\perp} \sin \theta$ and $\sigma_{\|}$are much less than the radiation wavelength. Measurement of the energy spectra of the transition radiation provides a method for obtaining information about the spatial and temporal distributions of the electron beam.

As (4) indicates, the finite transverse extent of the plasma produces a wavelength dependence in the differential energy for fully-coherent radiation. The spectral content of the radiation is no longer constant, as is the case for transition radiation from a fully-coherent beam traversing a boundary of infinite transverse extent, and the spectra is strongly modified by the diffraction effects for parameters $b \sim 1$ (i.e., $\lambda \sim$ $\rho / \gamma)$. Distortion of the spectra increases with larger energy, and the spectra are suppressed for decreasing transverse size. For large transverse size $(b \gg 1)$, the fully-coherent spectra becomes constant (i.e., the limit of transition radiation from an infinite transverse boundary). In general, the spectral region of coherent radiation is approximately $2 \pi \sigma_{z}<\lambda<2 \pi \rho / u$, where the lower bound is due to longitudinal coherence $[F$ in (4)], and the upper bound is due to diffraction effects $[D$ in (4)].

Using (4), assuming $b \gg 1$, the total coherent radiation energy into a small collection angle $\theta \leq \theta_{\text {coll }}<1 /\langle u\rangle$, and for a bandwidth $\Delta \omega / \omega$ is

$$
W_{\mathrm{CTR}} \simeq m_{e} c^{2}\left(r_{e} / \lambda\right) N_{b}^{2}(\Delta \omega / \omega) \theta_{\text {coll }}^{4}\left\langle u^{2}\right\rangle^{2},
$$

assuming $\left\langle u^{2}\right\rangle>1$. Laser-plasma generated electron beams in the self-modulated regime can be characterized by a Boltzmann momentum distribution $g(u)=u_{\mathrm{T}}^{-1} \exp \left[-u / u_{\mathrm{T}}\right]$, where $u_{\mathrm{T}}$ is the temperature of the distribution. The amount of radiated energy in the collection cone half-angle $\theta_{\text {coll }}<$ $1 / u_{\mathrm{T}}<1$ and frequency bandwidth $\Delta \omega / \omega$ is given by $\Delta W_{\mathrm{CTR}} \simeq 4 m_{e} c^{2}\left(r_{e} / \lambda\right) N_{b}^{2}\left(u_{\mathrm{T}} \theta_{\text {coll }}\right)^{4}(\Delta \omega / \omega)$. For example, $\Delta W_{\mathrm{CTR}}=30 \mu \mathrm{J}$ within $\theta_{\text {coll }}=50 \mathrm{mrad}$ for a $1.5 \mathrm{nC}$ bunch with temperature $u_{\mathrm{T}}=9$ in a bandwidth 0.3 to $3 \mathrm{THz}$. Due to the strong dependence on electron energy and angle, $W_{\mathrm{CTR}} \propto\left(\gamma \theta_{\text {coll }}\right)^{4}$, the measured energy can easily be increased by increasing either the electron energy $\gamma$ or the cone angle $\theta_{\text {coll }}$ of the collection optics (for $\theta_{\text {coll }}<1 /\langle u\rangle$ ).

\section{B. Experiment}

The experiments described in this paper used the short pulse, high peak power and high repetition rate $\mathrm{Ti}: \mathrm{Al}_{2} \mathrm{O}_{3}$ laser system [18] of the L'OASIS facility at LBNL. Low energy laser pulses (of wavelength $\lambda \simeq 0.8 \mu \mathrm{m}$ ) from a Ti: $\mathrm{Al}_{2} \mathrm{O}_{3}$ laser oscillator were first temporally stretched, amplified to $1 \mathrm{~J} /$ pulse level, and then compressed using a grating based optical compressor. Following compression, the laser beam was focused to a spot size $w=6 \mu \mathrm{m}$ with a $30 \mathrm{~cm}$ focal length (F/4) off-axis parabola onto a pulsed gas jet. The peak power $P$ of the laser was varied using both the pulse duration and laser energy. At optimum compression [55 fs full-width-half-maximum (FWHM) duration], $P \simeq 8.3 \mathrm{TW}$, resulting in a calculated peak intensity $I=2 P / \pi r_{s}^{2} \simeq$ $1.5 \times 10^{19} \mathrm{~W} / \mathrm{cm}^{2}$ and a normalized laser strength $a_{0} \simeq$ $8.6 \times 10^{-10} \lambda[\mu \mathrm{m}] I^{1 / 2}\left[\mathrm{~W} / \mathrm{cm}^{2}\right] \simeq 2.6$. The gas jet was backed with up to 70 bar helium gas. The profile of the jet had a $1.8 \mathrm{~mm}$ flat top at a density of about $3 \times 10^{19} \mathrm{~cm}^{-3}$ with a ramp of $1 \mathrm{~mm}$ length to zero density on either side (a total length of just under $4 \mathrm{~mm}$ ). The plasma density profile, measured with a folded-wave interferometer using $400 \mathrm{~nm}$ wavelength, $50 \mathrm{fs}$ duration laser pulses, had a typical transverse size of $100 \mu \mathrm{m} \pm 15 \mu \mathrm{m}$. The lay-out of the experiment in shown in Fig. 1 . 


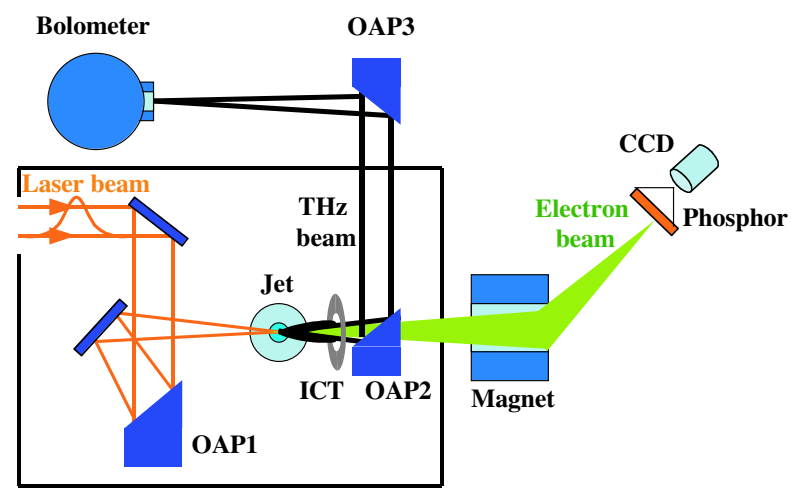

Fig. 1. Schematic of the experimental set-up. The high power laser beam is focused using an off-axis parabolic mirror (OAP1) onto a high pressure pulsed gas jet operating with about 70 bar He backing pressure. An integrating current transformer (ICT) is used to measure the charge per bunch of the electron beam produced in the laser ionized gas jet. A double focusing magnetic spectrometer is used to measure the electron beam energy by scanning the magnet excitation current. Radiation is collected with the use of a second off-axis parabola (OAP2) with a hole in the middle to transmit the high power laser beam and electron beam. OAP2 collimates the radiation which is transmitted through a silicon window and then focused using OAP3 onto the bolometer. This optical system collects radiation with angular direction between $80 \mathrm{mrad}$ and $300 \mathrm{mrad}$. The measurements of the radiated energy in a $30 \mathrm{mrad}$ solid angle were done by focusing the radiation onto the bolometer using a metal coated spherical mirror with a $50 \mathrm{~cm}$ focal length, located about $1 \mathrm{~m}$ away.

The charge per bunch and electron beam spatial profile were measured using an integrating current transformer (ICT) and a phosphor screen, located $50 \mathrm{~cm}$ and $75 \mathrm{~cm}$ away from the exit of the gas jet, respectively. The response of the phosphor screen (number of counts on the CCD vs. deposited charge) was calibrated against the ICT. The energy distribution was obtained using an imaging magnetic spectrometer [42] and a phosphor screen with CCD camera. At a given excitation current of the magnet, the momentum acceptance of the magnetic spectrometer was $\delta p / p= \pm 2 \%$. A spectrum ranging from $0-50 \mathrm{MeV}$ was obtained by recording the total light yield on the CCD image (corrected for background counts) for excitation currents ranging from 0 to $45 \mathrm{~A}$.

\section{Electron Beam Properties}

The present experiments were operated using a single laser pulse to ionize the gas jet and excite a plasma wake that subsequently traps and accelerates background electrons. For the particular gas jet density profile (absolute gas density and length of the jet) and laser parameters used in the present experiments, a typical energy spectrum is shown in Fig. 2 and is reasonably well approximated by a Boltzmann distribution with a temperature of $4.6 \mathrm{MeV}$.

The electron beam profile was found to be dependent on laser power. Two typical images of the electron beam on the phosphor screen in the accelerator operating regime for which the data were taken are shown in Fig. 3. The beam divergence vs. laser pulse duration is shown in Fig. 4. The laser pulse duration was changed by changing the distance between the gratings in the pulse compressor. The asymmetry in the data curve for points on the left and on the right hand side of the minimum pulse duration is consistent with previous

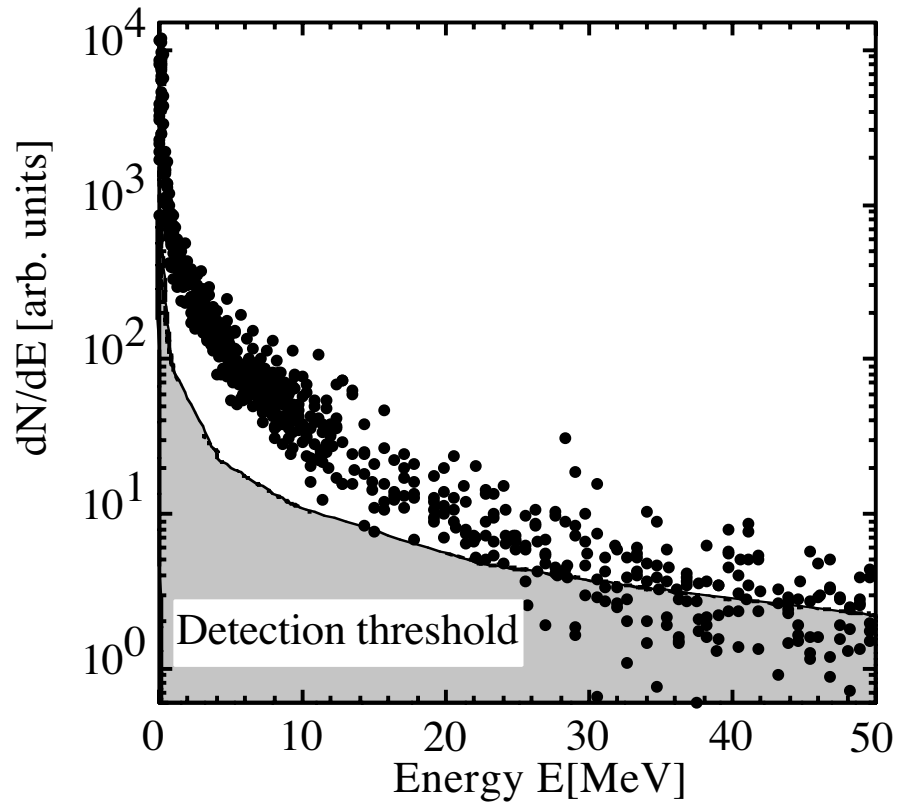

Fig. 2. Electron energy spectrum $d N / d E$ measured using a double focusing imaging magnetic spectrometer. The spectrum was obtained by scanning the excitation current in the magnet and measuring the intensity on a phosphor screen. For any given current, the momentum acceptance $\delta p / p$ of the spectrometer was $\pm 2 \%$. Each data point represents 10 shots. The spectrum is reasonably well approximated by a Boltzmann distribution with an effective temperature of $4.6 \mathrm{MeV}$.
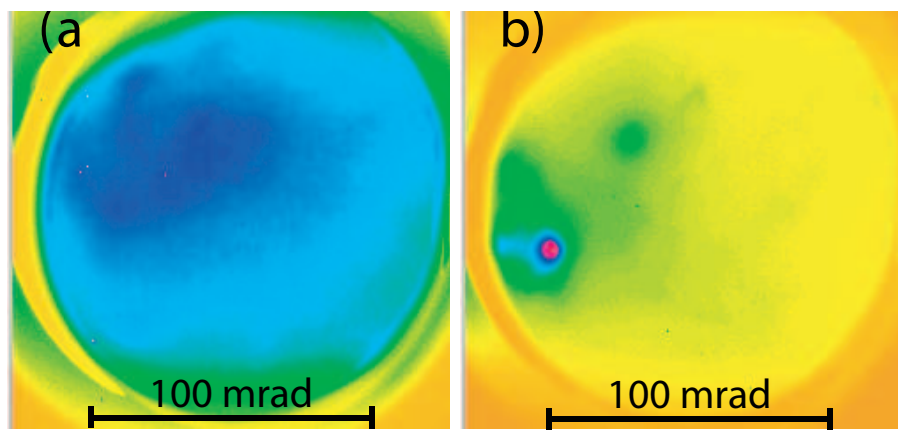

Fig. 3. Electron beam images measured by imaging a $10 \mathrm{~cm}$ diameter phosphor screen located $75 \mathrm{~cm}$ away from the gas jet with a CCD camera, for laser peak power (a) around 2-4 TW and (b) around $8 \mathrm{TW}$. The unnormalized geometric rms "emittance" for the small spot is $0.01 \mathrm{~mm}$-mrad, obtained by multiplying the assumed source size from which the beam originates $(6 \mu \mathrm{m}$ FWHM, i.e., equal to laser spot size) and a $10 \mathrm{mrad}$ (FWHM) divergence angle.

observations of a dependence of electron yield on the shape of the laser pulse. [7], [43]

For power levels near the maximum power, electron beams were observed with a divergence less than $10 \mathrm{mrad}$ (FWHM) [see Fig. 3(b)]. The amount of charge contained in the small beams was typically about $5 \%$ of the main beam and ranged between 30-50 pC. The energy of this part of the beam was not measured separately. However, the shot-to-shot fluctuation in beam divergence near maximum power was found to be significantly larger than for the lower power cases, as can be seen in Fig. 4 from the relatively larger error bars. In addition, the pointing varied from shot-to-shot by more than $20 \mathrm{mrad}$. 


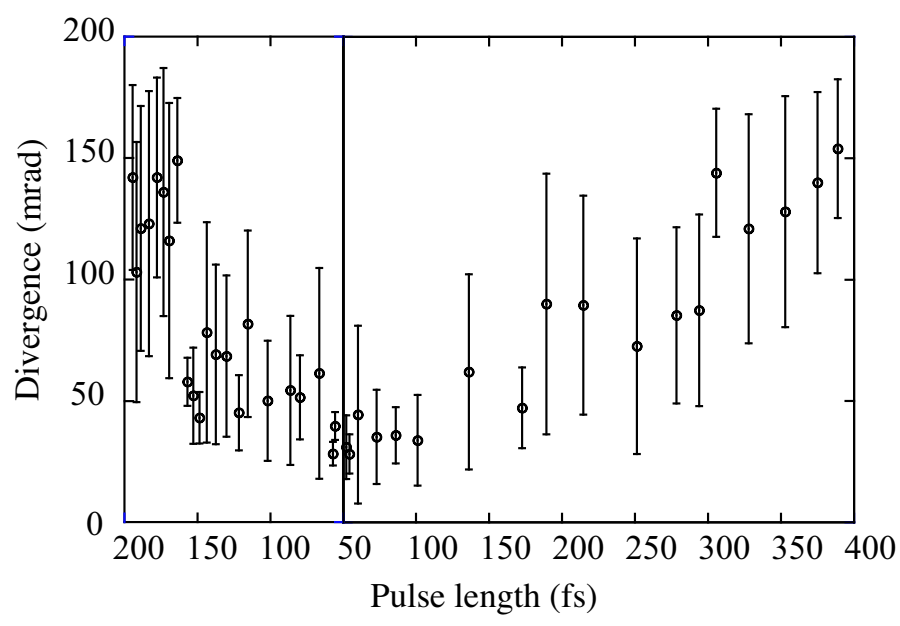

Fig. 4. Electron beam divergence vs. laser pulse duration. The laser pulse duration was varied by changing the distance between the gratings in the pulse compressor and the duration was measured using a single-shot second order autocorrelator. Each data point represents 10 shots.

For power levels which exceed the critical power for selffocusing by a factor of 5 or more, the laser self-channeling and particle trapping processes operate in a highly non-linear regime, which likely results in a loss of reproducibility due to sensitivity to operating parameters. In addition, pre-pulse effects have been shown to be important in the acceleration process and the production of stable beams [44].

Assuming that the electron beam originated from a spot size equal to the laser spot size $w$, a value for an unnormalized rms geometric "emittance" for the high power case can be estimated to be $0.01 \mathrm{~mm}$ mrad, by simply multiplying the assumed source size and measured divergence angle. Similarly low unnormalized "emittance" values between 0.01 and $0.1 \pi \mathrm{mm}$ mrad have been reported previously [44], [45]. However, prior to interpreting this quantity into an electron beam emittance value, as commonly used for conventional accelerators, a thorough understanding of all phase space properties of the beam is required, including energy (mean and spread) and bunch length, as a function of propagation distance. Upon exiting the plasma, the high density electron bunch is subjected to significant space charge forces that can result in transverse beam blow-up as well as modifications to the longitudinal bunch shape and energy distribution. In general, longitudinal space charge forces are expected to lengthen the bunch, thereby reducing the density and, consequently, the effect of transverse space charge forces while the beam is propagating through vacuum [46], [47]. Also, the large energy spread can result in ballistic spreading of the electron bunches since fast electrons would out run the slower ones. More complicated modifications of the energy distribution and correlations in momentum and physical space can still occur however, complicating the interpretation of the data. As an example, if the bunch initially has slow electrons in the front and back, and fast electrons in the middle, bunch compression can occur during the propagation resulting in a temporary increase in space charge forces. Detailed measurements of the energy (mean and spread), bunch length, and transverse characteristics of these low divergence beams are needed to fully understand their behavior during propagation in vacuum and to properly assign an emittance value. Pepper pot based measurements of electrons that were energy filtered around $55 \mathrm{MeV}$ have recently been carried out in a similar laser wakefield setup to ours and indicate a normalized emittance of $3 \pi \mathrm{mm}$ mrad at the spectrometer location [48].

\section{Radiation Measurements}

The radiation measurements were done from $0.3-19 \mathrm{THz}$ using a liquid helium cooled bolometer. To verify the dependence of radiated energy on collection angle, two different set-ups were used. In the first set-up, with a collection angle of $30 \mathrm{mrad}$, radiation was reflected out of the vacuum chamber through a window using a $5 \mu \mathrm{m}$ thick metal coated nitrocellulose foil $30 \mathrm{~cm}$ from the gas jet. (Although transition radiation will be generated when the beam propagates through this foil, the emission is incoherent due to bunch lengthening [47] and transverse beam size increase at that location, measured with a phosphor screen.) The radiation then propagated in air and through laser beam attenuators, and was focused with an F/30 metal coated spherical mirror onto a cooled $(4.2 \mathrm{~K}) \mathrm{Si}$ based bolometer, equipped with an internal $3 \mathrm{THz}$ low-pass filter, to measure the radiated energy. The spectral transmission properties of all solid materials used in the terahertz path were measured using a ZnTe based optical rectification system [49].

To improve collection efficiency, the second set-up used an off-axis $90^{\circ}$ parabola (effective focal length of $127 \mathrm{~mm}$ ) to collimate $\mathrm{THz}$ light onto a second off-axis parabola (effective focal length of $177.8 \mathrm{~mm}$ ). After exiting the vacuum chamber through a silicon window, the radiation was incident on the bolometer which was positioned in the calculated focal position. Note that a hole was drilled on axis through the first parabola to allow the $800 \mathrm{~nm}$ laser beam to propagate through it, resulting in a collection angle (half-cone) covering emission angles between $80<\theta<300 \mathrm{mrad}$. The complete bolometer system sensitivity (volts per incident energy) including a gain factor of the internal amplifier of 1000 and the bolometer internal collection efficiency was calibrated and found to be $1 \mathrm{~V}$ of signal per $4.0 \mathrm{pJ}$ of incident $\mathrm{THz}$ energy. The total transmission of all materials in the $\mathrm{THz}$ beam path was measured by a far-infrared interferometer in combination with a hot $\mathrm{Hg}$ lamp and found to be $2.5 \times 10^{-5}$.

For $\theta_{\text {coll }}=30 \mathrm{mrad}$, and electron beams containing up to $1.5 \mathrm{nC}$, the measured radiated energy per pulse between 0.3-2.9 THz was between 3-5 nJ/pulse [20] in the collection angle. For the set-up with collection angle covering 80$300 \mathrm{mrad}$ angles, the total radiated energy in the $\mathrm{THz}$ beam was approximately 70-80 $\mathrm{nJ}$ for electron bunches containing about $0.3 \mathrm{nC}$.

For the experimental parameters $(\rho=100 \mu \mathrm{m} \pm 15 \mu \mathrm{m}$, $\theta_{\text {coll }}=30 \mathrm{mrad}(+0 \mathrm{mrad} /-10 \mathrm{mrad}), 1.5 \mathrm{nC}$ charge, and $\left.u_{\mathrm{T}}=9(4.6 \mathrm{MeV})\right)$ the calculated energy per pulse is $2.3 \mathrm{~nJ}$, which agrees within error bars with the 3-5 nJ measured experimentally. For the second case, using $0.3 \mathrm{nC}$ charge, frequencies ranging from 0.3 to $19 \mathrm{THz}$, and angular coverage from $80-300 \mathrm{mrad}$, the calculated energy per pulse is $160 \mathrm{~nJ}$, 


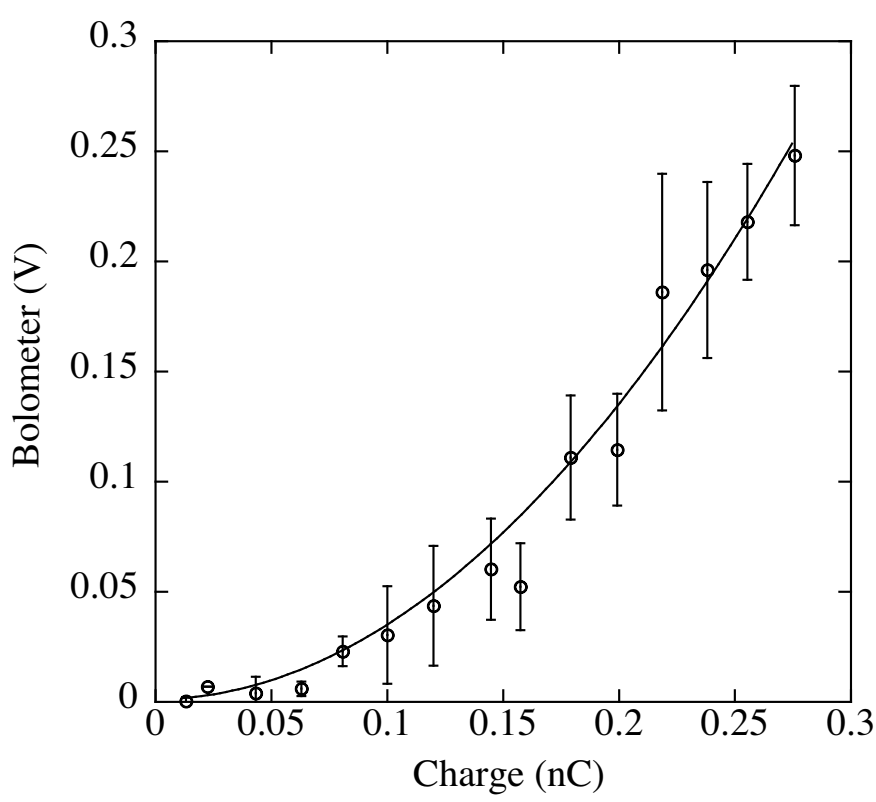

Fig. 5. Bolometer voltage vs. charge per bunch for the $80-300 \mathrm{mrad}$ collection half-angle. Each dot represents an average of 50 experimental shots, with rms variation as error bar. The solid line is a quadratic fit to the data, indicating coherence of the radiated signal. The bolometer (Infrared Laboratories) had a voltage responsivity $S=2.73 \times 10^{8} \mathrm{~V} / \mathrm{W}$ and thermal conductivity $G=17.01 \mu \mathrm{W} / \mathrm{K}$. Since the radiation bursts are much shorter than the bolometer time constant, $\tau_{\text {bolo }}=0.33 \mathrm{~ms}$, the detector operates as a calorimeter. The absorbed energy is then given by: $E_{\text {absorbed }}=G \tau_{\text {bolo }} \Delta V_{\text {bolo }} /\left(R \eta_{\text {bolo }}\right)=4.0 \times \Delta V_{\text {bolo }}$ (in pJ), where $R=S \times G=4.64$ is the induced voltage per $\mathrm{K}$ of temperature rise (in $\mathrm{V} / \mathrm{K}$ ), $\Delta V_{\mathrm{bolo}}$ is the height of the voltage pulse (in $\mathrm{V}$ ), and $\eta_{\mathrm{bolo}} \sim 0.3$ is the bolometer efficiency. The radiated energy is $E_{\text {radiated }}=E_{\text {absorbed }} / \eta_{\text {coll }}$, where $\eta_{\text {coll }}$ is the overall collection efficiency of the system.

compared to $70-80 \mathrm{~nJ}$ measured experimentally. This is in reasonable agreement, given the sensitivity of the radiated energy to bunch length, plasma size, energy distribution and total charge.

To verify that the radiation is emitted coherently, the bunch charge was varied by changing either the laser pulse duration or the position of the gas jet with respect to the laser beam focal position [7], [18]. The dependence of the peak amplitude of the bolometer signal versus bunch charge is shown in Fig. 5 and scales quadratically with charge, consistent with coherent emission of radiation.

\section{E. Polarization Measurement}

Since transition radiation is in principle radially polarized for an axisymmetric e-beam, a further test of the validity of our model was carried out by measuring the polarization characteristics of the source. A far-infrared wire-grid linear polarizer was placed at the entrance plane of the bolometer, where the $\mathrm{THz}$ radiation is brought to a focus. The polarizer angle was rotated from $0^{\circ}-180^{\circ}$ in steps of $30^{\circ}$ and at each position about 30 shots were acquired. Within the error bars, the transmitted signal was found to be independent of the polarizer angle, consistent with radially polarized radiation.

\section{F. Radiation Spectrum}

As a first attempt to obtain spectral information of the $\mathrm{THz}$ source, the total radiated energy was collected in the 0.3-19 $\mathrm{THz}$ and in the $0.3-2.9 \mathrm{THz}$ ranges (and hence $2.9-19 \mathrm{THz}$ range). This was done with the use of two separate filters that were inserted between the bolometer window and the bolometer detector element. The first long pass filter consisted of a thin polyethylene filter with a diamond scattering layer, and had a cut-on wavelength of $16 \mu \mathrm{m}(19 \mathrm{THz})$. The second long pass filter, made of crystal quartz with a broadband anti-reflection coating, had a cut-on wavelength of $103 \mu \mathrm{m}(2.9 \mathrm{THz})$. Radiation at wavelengths longer than $1 \mathrm{~mm}$ $(0.3 \mathrm{THz})$ was not detectable with the bolometer through its intrinsic design.

Analysis of the raw experimental data (averaging of several tens of shots) shows that $22 \%$ of the observed radiation was emitted in the frequency range $0.3-2.9 \mathrm{THz}$, while $78 \%$ of the radiation was emitted within the frequency range 2.9$19 \mathrm{THz}$ range. To model this data, the radiated spectrum was calculated including diffraction (see Sec. II-A) from a $100 \mu \mathrm{m}$ radius plasma column and again using a $4.6 \mathrm{MeV}$ Boltzmann distribution for the electron bunch energy distribution. The spectrum of the radiation can be calculated from (4) assuming a longitudinal charge distribution and transverse size of the dielectric (plasma). Figure 6 shows the calculated spectrum of the radiation for a Gaussian longitudinal charge distribution with rms length $\sigma_{z}=13.5 \mu \mathrm{m}$ and $u_{\mathrm{T}}=9$, transitioning out of a plasma with a transverse half-width of $100 \mu \mathrm{m}$. The short wavelength cut-off is due to the longitudinal coherence effects ( $\lambda_{\min } \sim \sigma_{z}$ ), and the long wavelength cut-off is due to the influence of diffraction radiation from the transverse edge of the plasma $\left(\lambda_{\max } \sim \rho / u_{\mathrm{T}}\right)$. For these values of plasma size and electron beam properties, the spectrum shown in Fig. 6 contains $22 \%$ of the radiation emitted in the frequency range 0.3 to $2.9 \mathrm{THz}$, and $78 \%$ of the radiation emitted within the frequency range 2.9 to $19 \mathrm{THz}$ range. An rms bunch length on the order of $10 \mu \mathrm{m}$ is consistent with self-modulated LWFA simulations [50] which typically predict electron beam bunch lengths to be on the order of the laser pulse duration.

\section{Femtosecond X-RAy Generation}

The ultra-short pulse nature of the accelerated electron bunches produced by a laser-plasma accelerator offers the possibility of generating femtosecond $\mathrm{x}$-ray pulses. Two different methods will be considered. The first relies on the emission of betatron radiation as the electrons propagate through a plasma channel in the presence of the radial wakefield excited by the laser pulse. The second method relies on the use of an external laser to Thomson scatter off the accelerated electron bunch.

\section{A. Betatron Radiation from Plasma Focusing Channels}

Betatron radiation will be analyzed starting with the single particle orbits to calculate the radiation spectrum. The on-axis radiation spectrum is calculated, as well as the asymptotic behavior of the spectrum for large values of the betatron strength parameter. Examples of the radiated spectrum are given for a single electron as well as for an electron beam with a Guassian radial distribution of electrons. 


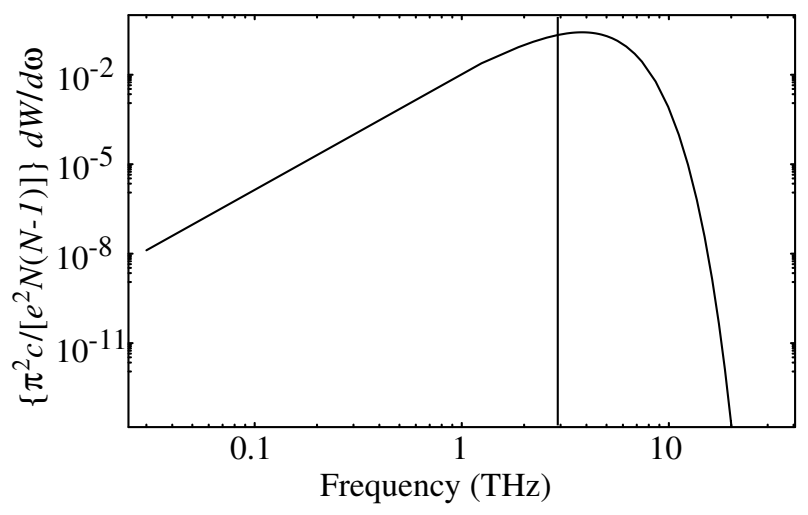

Fig. 6. Calculated radiated energy vs. frequency within $80<\theta_{\text {coll }}<$ $300 \mathrm{mrad}$. The measured electron beam energy distribution was approximated using a Boltzmann distribution with a temperature of $4.6 \mathrm{MeV}$, the longitudinal electron beam distribution was assumed to be a Gaussian with $13.5 \mu \mathrm{m} \mathrm{rms}$ length. and the plasma to have a transverse half-width $=100 \mu \mathrm{m}$. For these parameters, $22 \%$ of the radiated energy is emitted for frequencies $<2.9 \mathrm{THz}$ and $78 \%$ of energy for frequencies $>2.9 \mathrm{THz}$, as measured in the experiment.

1) Single Particle Orbits: The electron motion in a plasma focusing channel is governed by the relativistic Lorentz equation, which may be written in the form $d \mathbf{u} / d c t=\nabla \hat{\Phi}$, where $\hat{\Phi}=e \Phi / m_{e} c^{2}$ is the normalized electrostatic potential of the focusing channel, $\mathbf{u}=\mathbf{p} / m_{e} c=\gamma \beta$ is the normalized electron momentum, and $\gamma=\left(1+u^{2}\right)^{1 / 2}=\left(1-\beta^{2}\right)^{-1 / 2}$ is the relativistic factor. Here only the transverse focusing force of the plasma is considered. Near the axis, $r^{2} \ll$ $r_{0}^{2}$, the space charge potential is assumed to have the form $\hat{\Phi}=\hat{\Phi}_{0}\left(1-r^{2} / r_{0}^{2}\right)$, such that the normalized radial electric field is $\hat{E}_{r}=-\partial \hat{\Phi} / \partial r=2 \hat{\Phi}_{0} r / r_{0}^{2}$, where $\hat{\Phi}_{0}$ and $r_{0}$ are constants. The electrostatic potential is related to the electron plasma density by $\nabla^{2} \hat{\Phi}=k_{p}^{2}\left(n_{e} / n_{0}-1\right)$, where a uniform background of plasma ions of density $n_{0}$ is assumed. The maximum focusing field occurs when the plasma electrons are completely expelled (blown out) from the channel, $n_{e}=0$. Notice that in the blowout regime, $\hat{E}_{r}=k_{p}^{2} r / 2$, hence, $\hat{\Phi}_{0} / r_{0}^{2} \leq k_{p}^{2} / 4$. Assuming the electron orbit lies in the $(x, z)$ plane, the betatron orbit is given by

$$
\begin{gathered}
\tilde{\beta}_{x} \simeq k_{\beta} r_{\beta} \cos \left(k_{\beta} c t\right) \\
\tilde{x} \simeq r_{\beta} \sin \left(k_{\beta} c t\right), \\
\tilde{\beta}_{z} \simeq \beta_{z 0}\left(1-k_{\beta}^{2} r_{\beta}^{2} / 4\right)-\beta_{z 0}\left(k_{\beta}^{2} r_{\beta}^{2} / 4\right) \cos \left(2 k_{\beta} c t\right), \\
\tilde{z} \simeq z_{0}+\beta_{z 0}\left(1-k_{\beta}^{2} r_{\beta}^{2} / 4\right) c t-\beta_{z 0}\left(k_{\beta}^{2} r_{\beta}^{2} / 8\right) \sin \left(2 k_{\beta} c t\right),
\end{gathered}
$$

where $k_{\beta}=\left(2 \hat{\Phi}_{0} / \gamma_{z 0} r_{0}^{2}\right)^{1 / 2}$ is the betatron wavenumber, $r_{\beta}$ is the amplitude (assumed constant) of the betatron orbit, $u_{z}=u_{z 0}$ is the initial axial momentum of the electron (a constant of the motion), $\gamma_{z 0}=\left(1+u_{z 0}^{2}\right)^{1 / 2}, \beta_{z 0}=u_{z 0} / \gamma_{z 0}$ and $z_{0}$ is a constant. The above expressions are the leading order contributions to the orbits, assuming $k_{\beta}^{2} r_{\beta}^{2} / 2 \ll 1$. Notice that in the blowout regime $\hat{\Phi}_{0}=k_{p}^{2} r_{0}^{2} / 4$, which gives $k_{\beta}=k_{p} /\left(2 \gamma_{z 0}\right)^{1 / 2}$.

2) Single Particle Radiation Spectrum: The energy spectrum of the radiation emitted by a single electron on an arbitrary orbit $\tilde{\mathbf{r}}(t)$ and $\tilde{\beta}(t)$ can be calculated from the
Lienard-Wiechert potentials [51],

$$
\frac{d^{2} I}{d \omega d \Omega}=\frac{e^{2} \omega^{2}}{4 \pi^{2} c}\left|\int_{-T / 2}^{T / 2} d t[\mathbf{n} \times(\mathbf{n} \times \tilde{\beta})] e^{i \omega(t-\mathbf{n} \cdot \tilde{\mathbf{r}} / c)}\right|^{2}
$$

where $d^{2} I / d \omega d \Omega$ is the energy radiated per frequency, $\omega$, per solid angle, $\Omega$, during the interaction time, $T$, and $\mathbf{n}$ is a unit vector pointing in the direction of observation. Using the betatron orbits given above, the radiation spectrum can be calculated with conventional techniques [27], [52]. In the limits $\gamma_{z 0}^{2} \gg 1, \theta^{2} \ll 1$, and $\left(1+a_{\beta}^{2} / 2\right) / \gamma_{z 0}^{2} \ll 1$, the radiation spectrum can be written as

$$
\begin{aligned}
& \frac{d^{2} I}{d \hbar \omega}=\sum_{n=1}^{\infty} \frac{\alpha_{f} \gamma_{z 0}^{2} N_{\beta}^{2}\left(\omega^{2} / \omega_{n}^{2}\right)}{\left(1+a_{\beta}^{2}+\gamma_{z 0}^{2} \theta^{2}\right)^{2}} R_{n}\left(\omega, \omega_{n}\right) \\
& \quad \times\left[a_{\beta}^{2} \hat{C}_{x}^{2}+4 \gamma_{z 0}^{2} \theta^{2} \hat{C}_{z}^{2}-4 a_{\beta} \gamma_{z 0} \theta \hat{C}_{x} \hat{C}_{z} \cos \phi\right],
\end{aligned}
$$

where

$$
\begin{gathered}
\hat{C}_{x}=\sum_{m=-\infty}^{\infty} J_{m}\left(\alpha_{z}\right)\left[J_{n+2 m-1}\left(\alpha_{x}\right)+J_{n+2 m+1}\left(\alpha_{x}\right)\right] \\
\hat{C}_{z}=\sum_{m=-\infty}^{\infty} J_{m}\left(\alpha_{z}\right) J_{n+2 m}\left(\alpha_{x}\right) \\
\alpha_{z}=\frac{n\left(\omega / \omega_{n}\right) a_{\beta}^{2} / 4}{\left(1+a_{\beta}^{2} / 2+\gamma_{z 0}^{2} \theta^{2}\right)} \\
\alpha_{x}=\frac{2 n\left(\omega / \omega_{n}\right) a_{\beta} \gamma_{z 0} \theta \cos \phi}{\left(1+a_{\beta}^{2} / 2+\gamma_{z 0}^{2} \theta^{2}\right)}
\end{gathered}
$$

and

$$
R_{n}=\frac{\sin ^{2}\left[\pi n N_{\beta}\left(\omega / \omega_{n}-1\right)\right]}{\left[\pi n N_{\beta}\left(\omega / \omega_{n}-1\right)\right]^{2}}
$$

is the resonance function, with $\omega=c k$ is the radiation wavenumber, $\omega_{n}=c k_{n}$ is the resonant wavenumber for the $n^{\text {th }}$ harmonic, which is given by

$$
\omega_{n}=\frac{2 \gamma_{z 0}^{2} n c k_{\beta}}{\left(1+a_{\beta}^{2} / 2+\gamma_{z 0}^{2} \theta^{2}\right)},
$$

$n$ is the harmonic number,

$$
a_{\beta}=\gamma_{z 0} k_{\beta} r_{\beta}
$$

is the betatron strength parameter, $N_{\beta}=L / \lambda_{\beta}$ the number of betatron periods that the electron undergoes, $L=c T$ is the interaction length, $J_{m}$ are Bessel functions, and $\alpha_{f}=e^{2} / \hbar c \simeq$ $1 / 137$ the fine structure constant. Here $\theta$ is the observation angle with respect to the electron propagation axis and $\phi$ is the azimuthal observation angle.

The resonance function $R_{n}\left(\omega, \omega_{n}\right)$ determines many properties of the radiation spectrum. Provided the number of betatron periods is large, $N_{\beta} \gg 1$, radiation is emitted in a series of harmonics and is confined in a narrow bandwidth about the resonant frequency $\left(\omega=\omega_{n}\right)$ of each harmonic. The intrinsic frequency width $\Delta \omega_{n}$ of the spectrum $R_{n}$ about $\omega_{n}$ is given by $\Delta \omega_{n} / \omega_{n}=1 / n N_{\beta}$. Furthermore, $R_{n}\left(\omega, \omega_{n}\right) \rightarrow$ $\Delta \omega_{n} \delta\left(\omega-\omega_{n}\right)$ as $N_{\beta} \rightarrow \infty$. For a single harmonic $n$, the angular width $\Delta \theta_{I}$ about the axis of a cone containing 
radiation with frequencies in a small bandwidth $\Delta \omega$ about $\omega_{n}$ is given by

$$
\Delta \theta_{I}^{2} \simeq\left(\frac{1+a_{\beta}^{2} / 2}{\gamma_{z 0}^{2}}\right) \times \begin{cases}\Delta \omega_{n} / \omega_{n}, & \Delta \omega \leq \Delta \omega_{n} \\ \Delta \omega / \omega_{n}, & \Delta \omega \geq \Delta \omega_{n}\end{cases}
$$

3) On-Axis Radiation: Of particular interest is the radiation emitted along the axis, $\theta=0$, where only the odd harmonics are finite, i.e., the even harmonics vanish. Setting $\theta=0$ in the above expressions gives, for the $n^{\text {th }}$ odd harmonic, $\alpha_{x}=0$, $\alpha_{z}=\alpha_{n}$, and

$$
\begin{aligned}
\frac{d^{2} I_{n}(0)}{d \hbar \omega d \Omega}=\alpha_{f} & \left(\frac{\omega}{\omega_{n}}\right)^{2} \frac{\gamma_{z 0}^{2} N_{\beta}^{2} n^{2} a_{\beta}^{2}}{\left(1+a_{\beta}^{2} / 2\right)^{2}} R_{n}\left(\omega, \omega_{n}\right) \\
& \times\left[J_{(n-1) / 2}\left(\alpha_{n}\right)-J_{(n+1) / 2}\left(\alpha_{n}\right)\right]^{2},
\end{aligned}
$$

where $\alpha_{n}=n\left(\omega / \omega_{n}\right)\left(a_{\beta}^{2} / 4\right) /\left(1+a_{\beta}^{2} / 2\right)$ and $\omega_{n}=$ $2 n \gamma_{z 0}^{2} c k_{\beta} /\left(1+a_{\beta}^{2} / 2\right)$ is the on-axis resonant frequency.

An expression for the number of photons $\left(N_{n}\right)$ radiated along the axis per solid angle, $d N_{n} / d \Omega$, per electron for photons in a narrow bandwidth $\Delta \omega$ about the resonant frequency $\omega_{n}$ is obtained by integrating the above expression over $\Delta \omega$ and by dividing by the energy per photon $\left(\hbar \omega_{n}\right)$,

$$
\begin{aligned}
\frac{d N_{n}}{d \Omega} \simeq \alpha_{f} \frac{\Delta \omega_{I}}{\omega_{n}} & \frac{\gamma_{z 0}^{2} N_{\beta}^{2} n^{2} a_{\beta}^{2}}{\left(1+a_{\beta}^{2} / 2\right)^{2}} \\
& \times\left[J_{(n-1) / 2}\left(\alpha_{n}\right)-J_{(n+1) / 2}\left(\alpha_{n}\right)\right]^{2},
\end{aligned}
$$

where $\Delta \omega_{I}=\Delta \omega$ for $\Delta \omega \leq \Delta \omega_{n}$ and $\Delta \omega_{I}=\Delta \omega_{n}$ for $\Delta \omega \geq \Delta \omega_{n}$, with $\Delta \omega_{n}=\omega_{n} / n N_{\beta}$ the intrinsic bandwidth and $\alpha_{f}$ the fine structure constant. The total number of photons radiated per electron in the bandwidth $\Delta \omega$ about $\omega_{n}$ is given by multiplying $d N_{n} / \delta \Omega$ by the solid angle $2 \pi\left(\Delta \theta_{I}^{2} / 2\right)^{1 / 2}$, where $\Delta \theta_{I}$ is given above. This yields

$N_{n} \simeq \pi \alpha_{f} \frac{\Delta \omega}{\omega_{n}} \frac{N_{\beta} n a_{\beta}^{2}}{\left(1+a_{\beta}^{2} / 2\right)}\left[J_{(n-1) / 2}\left(\alpha_{n}\right)-J_{(n+1) / 2}\left(\alpha_{n}\right)\right]^{2}$,

for all values of $\Delta \omega^{2} \ll \omega_{n}^{2}$.

In the limit $a_{\beta}^{2} \ll 1$, this reduces to $N_{n} \simeq$ $\pi \alpha_{f}\left(\Delta \omega / \omega_{n}\right) N_{\beta} a_{\beta}^{2}$. By integrating the full expression for $d^{2} I / d \omega d \Omega$ over all frequencies and angles, one finds that the total number of photons radiated is given by

$$
N_{T}=(\pi / 3) \alpha_{f} N_{\beta} a_{\beta}^{2},
$$

assuming $a_{\beta}^{2} \ll 1$.

4) Asymptotic Behavior: For values of $a_{\beta}^{2} \ll 1$, the emitted radiation will be narrowly peaked about the fundamental resonant frequency, $\omega_{1}(n=1)$. As $a_{\beta}$ approaches unity, emitted radiation will appear at harmonics of the resonant frequency as well, $\omega_{n}=n \omega_{1}$. When $a_{\beta} \gg 1$, high harmonic $(n \gg 1)$ radiation is generated and the resulting synchrotron radiation spectrum consists of many closely spaced harmonics. Finite variations in the parameter $a_{\beta}=\gamma_{z 0} k_{\beta} r_{\beta}$ within an electron beam can broaden the linewidth and cause the spectrum to overlap. Hence, in the asymptotic limit, i.e., $a_{\beta} \gg 1$, the gross spectrum appears broadband, and a continuum of radiation is generated which extends out to a critical frequency, $\omega_{c}$, beyond which the radiation intensity diminishes.
Asymptotic properties of the radiation spectrum for $a_{\beta}^{2} \gg 1$ and for large harmonic numbers, $n \gg 1$, can be obtained with standard methods. In particular, the asymptotic spectrum along the axis $\theta=0$, is given by

$$
\frac{d^{2} I(0)}{d \hbar \omega d \Omega} \simeq\left(6 / \pi^{2}\right) \alpha_{f} N_{\beta} \gamma_{z 0}^{2} \xi^{2} K_{2 / 3}^{2}(\xi),
$$

where $\xi=\omega / \omega_{c}$ and $\omega_{c} \simeq 3 a_{\beta} \gamma_{z 0}^{2} c k_{\beta}$ is the critical frequency (corresponding to a critical harmonic number of $n_{c}=3 a_{\beta}^{3} / 4$ ). The function $Y(\xi)=\xi^{2} K_{2 / 3}^{2}(\xi)$ is maximum at $\xi=1 / 2$ and decreases rapidly for $\xi>1$. Half the total power is radiated at frequencies $\omega<\omega_{c} / 2$ and half at $\omega>\omega_{c} / 2$.

5) Radiation from a Beam: For a single electron undergoing betatron motion in a plasma focusing channel, the resonant frequency of the radiation emitted along the axis is $\omega=$ $2 \gamma_{z 0}^{2} n \omega_{\beta} /\left(1+a_{\beta}^{2} / 2\right)$. Here, $a_{\beta}=\gamma_{z 0} k_{\beta} r_{\beta}$ is a function of both the electron energy $\gamma_{z 0}$ and the radial position of the electrons via the betatron amplitude $r_{\beta}$. If a monoenergetic beam of finite radius is injected into a focusing channel (without any special tapering), electrons at different radii will have different betatron amplitudes $r_{\beta}$, different values of $a_{\beta}$, and hence different resonant frequencies. In general, the spectral energy density of the radiation emitted by a finite radius beam will be significantly different from that of a single electron, especially in the limit $a_{\beta} \gtrsim 1$.

Consider the case of a monoenergetic, axisymmetric (round) beam in cylindrical geometry in the limit of zero emittance. In this case, $a_{\beta}=\gamma_{z 0} k_{\beta} r_{\beta}$ represents the normalized radial position of the electron, since the initial radial position of the electron at the channel entrance is assumed to be equal to $r_{\beta}$. The radiation spectrum from a beam, $d^{2} I_{B} / d \omega d \Omega$ can be approximately calculated from $d^{2} I / d \omega d \Omega$ by multiplying by the electron distribution function, $f_{e}$, and integrating over both radius $\left(a_{\beta}\right)$ and over $\phi$ (from 0 to $2 \pi$ for an axisymmetric beam). For an axisymmetric beam,

$$
\frac{d^{2} I_{B}(\omega, \theta)}{d \omega d \Omega}=\int_{0}^{2 \pi} \frac{d \phi}{2 \pi} \int_{0}^{\infty} d a_{\beta} a_{\beta} f_{e}\left(a_{\beta}\right) \frac{d^{2} I\left(\omega, a_{\beta}, \theta, \phi\right)}{d \omega d \Omega} .
$$

For simplicity, a Gaussian radial beam distribution $f_{e}\left(a_{\beta}\right)=$ $\left(2 / a_{\mathrm{rms}}^{2}\right) \exp \left(-a_{\beta}^{2} / a_{\mathrm{rms}}^{2}\right)$ is assumed, where $a_{\mathrm{rms}}=\gamma_{z 0} k_{\beta} r_{b}$ and $r_{b}$ is the normalized RMS beam radius.

For a large number of betatron periods, the radial integration can be approximated analytically. Let

$$
S_{R}=\int_{0}^{\infty} d a_{\beta} a_{\beta} f_{e} \frac{d^{2} I}{d \omega \delta \Omega} \equiv \int_{0}^{\infty} d a_{\beta} a_{\beta} f_{e} S_{n} R_{n}\left(\omega, a_{\beta}, \theta\right),
$$

where $R_{n}$ is the resonance function. At resonance $\omega=$ $\omega_{n}\left(a_{\beta}\right)$ or, alternatively, $a_{\beta}=a_{r}(\omega)$, where $a_{r}^{2}=$ $2\left[n 2 \gamma_{z 0}^{2} c k_{\beta} / \omega-\left(1+\gamma_{z 0}^{2} \theta^{2}\right)\right]$. Furthermore, in the limit $N_{\beta} \rightarrow \infty, R_{n} \rightarrow \Delta a_{r} \delta\left(a_{\beta}-a_{r}\right)$, where $\Delta a_{r}=$ $2 \gamma_{z 0}^{2} c k_{\beta} /\left(N_{\beta} a_{r} \omega\right)$. Hence, for $N_{\beta} \rightarrow \infty$,

$$
S_{R} \simeq \frac{2 \gamma_{z 0}^{2} c k_{\beta}}{N_{\beta} \omega} f_{e}\left(a_{\beta}=a_{r}\right) S_{n}\left(a_{\beta}=a_{r}\right) .
$$

In this limit, the spectrum of the radiation emitted along the 
axis $(\theta=0)$ from a Gaussian beam profile is

$$
\begin{aligned}
\frac{d^{2} I_{B}(0)}{d \hbar \omega d \Omega}=\sum_{n} & \alpha_{f} f_{e}\left(a_{r}\right) \frac{n \gamma_{z 0}^{2} N_{\beta} a_{r}^{2}}{\left(1+a_{r}^{2} / 2\right)} \\
& \times\left[J_{(n-1) / 2}\left(\alpha_{n}\right)-J_{(n+1) / 2}\left(\alpha_{n}\right)\right]^{2},
\end{aligned}
$$

where the sum is over odd harmonics $n$ and the argument of the Bessel functions is $\alpha_{n}=(n-\hat{\omega}) / 2$, where $\hat{\omega}=$ $\omega / 2 \gamma_{z 0}^{2} c k_{\beta}$, i.e., evaluated at $a_{\beta}^{2}=a_{r}^{2}=2(n / \hat{\omega}-1)$.

Using the above expression, other quantities of practical interest, such as the photon flux and brightness, can be calculated. Assuming that the collection angle, $\theta_{d}$, is small $\left(\theta_{d}<(\Delta \omega / \omega)^{1 / 2} / \gamma<(1 / N)^{1 / 2} / \gamma\right)$ so that the intensity distribution is flat over the solid angle $\Delta \Omega_{d}=\pi \theta_{d}^{2}$, the number of photons intercepted in a small bandwidth, $\Delta \omega$, about $\omega$ and solid angle, $\Delta \Omega_{d}$, for an electron bunch with $N_{b}$ electrons is

$$
N_{B}=N_{b} \frac{d^{2} I_{B}(0)}{d \hbar \omega d \Omega} \frac{\Delta \omega}{\omega} \pi \theta_{d}^{2} .
$$

The average flux in photons per second, $F_{\text {ave }}$, in the collection angle $\theta_{d}$ and with bandwidth $\Delta \omega$, is $N_{B}$ multiplied by the repetition rate, $f_{r e p}$, of the laser/electron beam, i.e., $F_{\text {ave }}=$ $N_{T} f_{\text {rep }}$. The average source brightness is given by

$$
B_{\text {ave }}=\frac{N_{B} f_{r e p}}{(2 \pi)^{2} r_{b}^{2} \theta_{d}^{2}}=\frac{N_{b}}{4 \pi r_{b}^{2}} \frac{d^{2} I_{B}(0)}{d \hbar \omega d \Omega} \frac{\Delta \omega}{\omega},
$$

where $r_{b}$ is the electron bunch radius. The peak flux and brightness are, respectively, $F_{p k}=F_{a v e} /\left(\tau_{x} f_{r e p}\right)$ and $B_{p k}=$ $B_{\text {ave }} /\left(\tau_{x} f_{\text {rep }}\right)$, where $\tau_{x}$ is the x-ray pulse duration, which is assumed to be approximately equal to the electron bunch duration.

The radiation spectra for a single electron with $a_{\beta}=2$ and from an axisymmetric beam with a Gaussian radial distribution with $a_{\mathrm{rms}}=2$ are shown in Figs. 7-8. The result for a single electron with $N_{\beta}=4$ and $a_{\beta}=2$, is shown in Fig. 7, which shows the spectral density $d^{2} I / d \hbar \omega d \Omega$ (normalized to $\alpha_{f} \gamma_{z 0}^{2}$ ) versus normalized frequency $\hat{\omega}=\omega / 2 \gamma_{z 0}^{2} c k_{\beta}$ and angle $\hat{\theta}=$ $\gamma_{z 0} \theta$, for (a) $\phi=0$ and (b) $\phi=\pi / 2$. The results of averaging over only $\phi$ for $a_{\beta}=2$ are shown in Fig. 8(a), whereas the results of averaging over both $\phi$ and $a_{\beta}$ are shown in Fig. 8(b). The effects of averaging over $a_{\beta}$ leads to a dramatic smoothing of the radiation spectrum.

As an example, consder a laser wakefield accelerator producing a narrow energy spread electron beam, such as in the proposed colliding pulse injection method [10], [12], [13], or as in the recent experiments on the channel-guided LWFA [15]. The high energy part of the electron spectrum is assumed to exhibit a narrow peak at $100 \mathrm{MeV}$ (3 percent energy spread) with a total charge of $0.3 \mathrm{nC}$ and a bunch radius of $3 \mu \mathrm{m}$. The plasma is assumed to be $1 \mathrm{~mm}$ long with a density of $2 \times$ $10^{19} \mathrm{~cm}^{-3}$, and the wakefield is assumed to be in the blowout regime. Note that for an electron with a betatron radius equal to the beam radius $(3 \mu \mathrm{m}), a_{\beta} \simeq 26$. The expected radiated spectrum for a detection angle of $\theta_{d}=3 \mathrm{mrad}$, normalized to a bandwidth of $\Delta \omega / \omega=0.1 \%$, is shown in Fig. 9, as obtained from (30). At the peak of the spectrum $(10 \mathrm{keV})$, the number of photons is $N_{B}=2 \times 10^{4}$ photons/shot and the brightness is
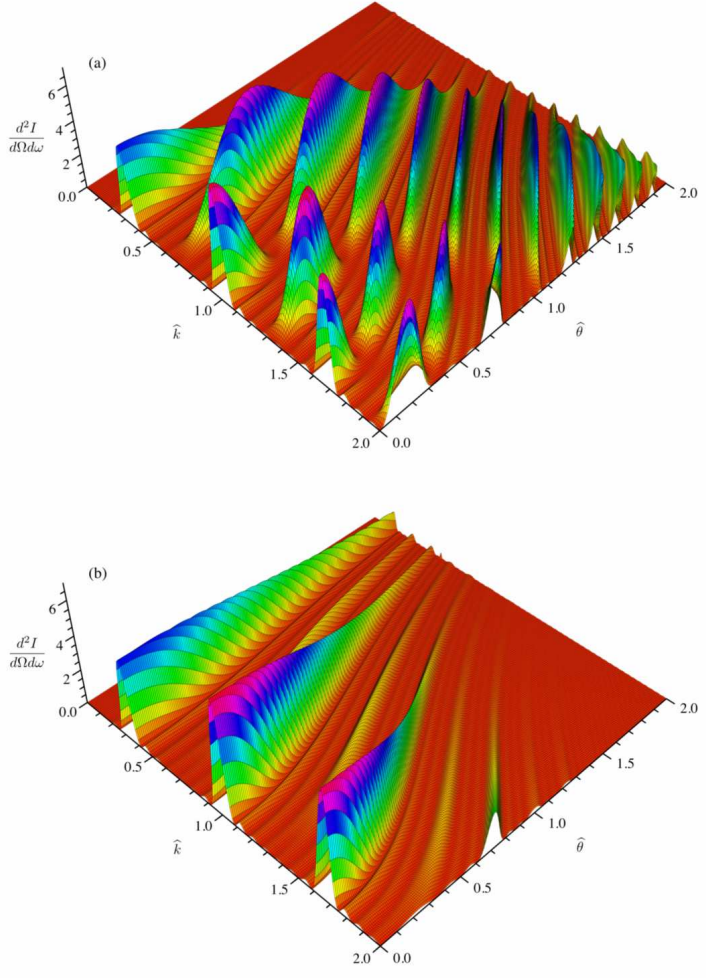

Fig. 7. Normalized spectrum $d^{2} I / d \omega \delta \Omega$ (arbitrary units) versus normalized frequency $\hat{k}=\hat{\omega} / c$ and angle $\hat{\theta}$ from a single electron with $a_{\beta}=2$ and $N_{\beta}=4$ for (a) $\phi=0$ and (b) $\phi=\pi / 2$.

$B_{\text {ave }}=6.3 \times 10^{6}$ photons $/$ shot $/ 0.1 \% \mathrm{BW} / \mathrm{mm}^{2} / \mathrm{mrad}^{2}$, assuming a 3 mrad detection angle and $0.1 \%$ bandwidth, as obtained from (31) and (32).

\section{B. Thomson Scattering using an External Laser Pulse}

The analysis of this $\mathrm{x}$-ray source will again start with a discussion on the orbit of a single electron and the spectrum will be calculated in a similar way as for betatron radiation. The scattered spectrum for a single electron will be calculated as well as that from an electron beam with finite energy spread. The obtained expressions will be used to design a polychromatic and quasi-monochromatic source, obtained by scattering off an electron bunch with a broad (100\%) and a narrow (few \%) energy distribution, respectively.

1) Single Particle Orbits: Consider a one-dimensional (1D) laser field propagating in the negative $z$ direction with a normalized vector potential, $a=e A / m_{e} c^{2}$, of the form $a=a_{0} \cos k_{0}(z+c t)$, which is linearly polarized in the $x$ direction with frequency $\omega_{0}=c k_{0}$. Here, the parameter $a_{0}$ is related to the laser intensity $I_{L}$ and wavelength $\lambda_{0}$ by $a_{0} \simeq 8.5 \times 10^{-10} \lambda[\mu \mathrm{m}]\left(I_{L}\left[\mathrm{~W} / \mathrm{cm}^{2}\right]\right)^{1 / 2}$. The electron motion is governed by the relativistic Lorentz equation,

$$
d \mathbf{u} / d c t=\partial \mathbf{a} / \partial c t-(\mathbf{u} / \gamma) \times(\nabla \times \mathbf{a}) .
$$

In the 1D limit, there exist two constants of the motion, $\mathbf{u}_{\perp}=$ $\mathbf{a}_{\perp}$ and $\gamma+u_{z}=\gamma_{z 0}+u_{z 0}=\gamma_{z 0}\left(1+\beta_{z 0}\right)$, where $u_{z 0}=$ 


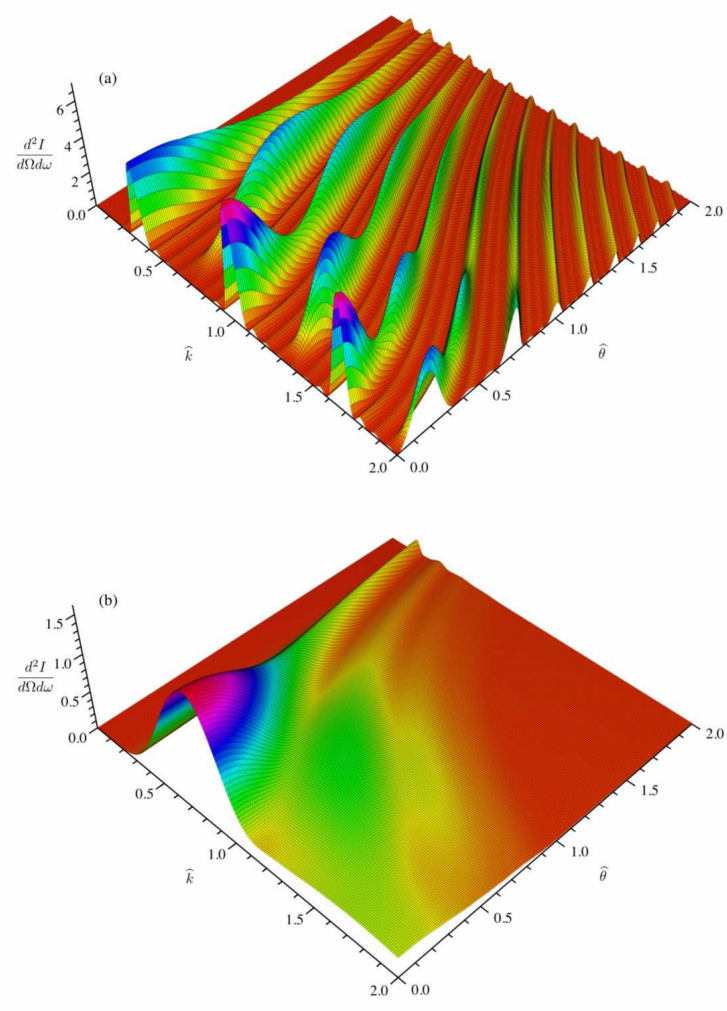

Fig. 8. Normalized spectrum $d^{2} I / d \omega \delta \Omega$ (arbitrary units) versus normalized frequency $\hat{k}=\hat{\omega} / c$ and angle $\hat{\theta}$ after (a) averaging over $\phi$ with $a_{\beta}=2$ and (b) averaging over both $\phi$ and $a_{\beta}$ with $a_{\mathrm{rms}}=2$ and $N_{\beta}=4$.

$\gamma_{z 0} \beta_{z 0}$ is the initial axial motion of the electron along the positive $z$ axis and $\gamma_{z 0}=\left(1+u_{z 0}^{2}\right)^{1 / 2}$. The two constants of the motion completely determine the electron orbits in the laser field. In particular,

$$
\begin{aligned}
& x(\eta)=x_{0}+\frac{a_{0} \sin k_{0} \eta}{k_{0} \gamma_{z 0}\left(1+\beta_{z 0}\right)}, \\
& z(\eta)=z_{0}+\left[\frac{\gamma_{z 0}^{2}\left(1+\beta_{z 0}^{2}\right)-\gamma_{\perp}^{2}}{2 \gamma_{z 0}^{2}\left(1+\beta_{z 0}\right)^{2}}\right] \eta-\frac{a_{0}^{2} \sin 2 k_{0} \eta}{8 k_{0} \gamma_{z 0}^{2}\left(1+\beta_{z 0}\right)^{2}}
\end{aligned}
$$

and $y(\eta)=y_{0}$, where $\eta=z+c t, \gamma_{\perp}^{2}=1+a_{0}^{2} / 2$, and $x_{0}, y_{0}$, and $z_{0}$ are initial conditions.

2) Scattered Spectrum for a Single Electron: With knowledge of the single particle orbits, the Thomson scattered radiation spectrum can be calculated in a manner similar to what was done for the case of betatron radiation, discussed in the previous section. In fact, in the limits $\gamma_{z 0}^{2} \gg 1, \theta^{2} \ll 1$, and $\gamma_{\perp}^{2} / \gamma_{z 0}^{2} \ll 1$, the radiation spectrum can be written in a form identical to (13) with the following substitutions. The betatron strength parameter $a_{\beta}$ is replaced by the laser strength parameter $a_{0}$, the number of betatron periods $N_{\beta}$ is replaced by the number of laser periods $N_{0}$, and the resonant frequency of each harmonics is given by

$$
\omega_{n}=\frac{4 \gamma_{z 0}^{2} n \omega_{0}}{\left(1+a_{0}^{2} / 2+\gamma_{z 0}^{2} \theta^{2}\right)} .
$$

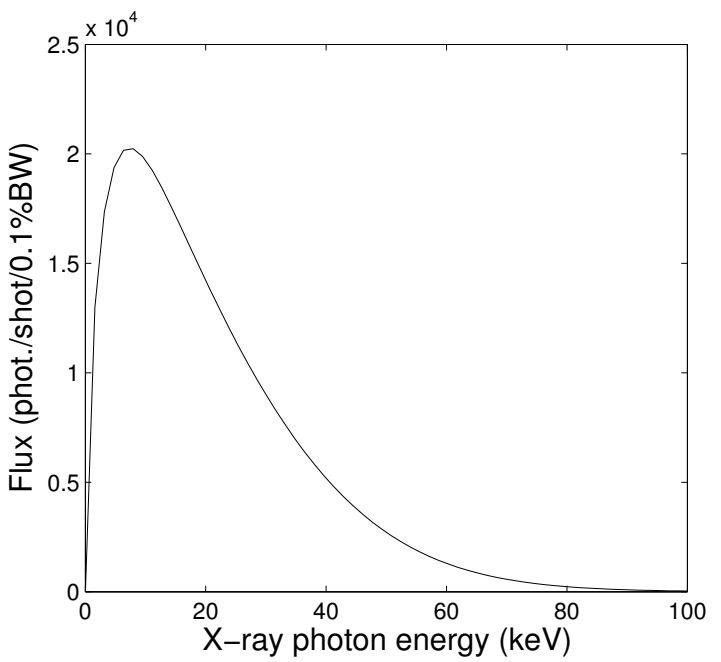

Fig. 9. Expected spectrum within a $3 \mathrm{mrad}$ detection angle from a $0.3 \mathrm{nC}$, $100 \mathrm{MeV}, 3 \mu \mathrm{m}$ radius electron beam undergoing betatron oscillations in a plasma channel with $1 \mathrm{~mm}$ length and $2 \times 10^{19} \mathrm{~cm}^{-3}$ electron density.

Likewise, the other expressions describing betatron radiation from a single electron also describe Thomson scattering, (21)(26), with the strength parameter replaced by $a_{0}$, the number of periods replaced by $N_{0}$, the resonant frequency replaced by (36), and the critical frequency replaced by $\omega_{c} \simeq 6 a_{0} \gamma_{z 0}^{2} \omega_{0}$. Similarly, it is noted that these same expressions also describe synchrotron radiation from an undulator or wiggler magnet in the limits $\gamma_{z 0}^{2} \gg 1, \theta^{2} \ll 1$, and $\left(1+a_{w}^{2} / 2\right) / \gamma_{z 0}^{2} \ll$ 1 , when the strength parameter is replaced by the wiggler strength parameter $a_{w}$, the number of periods replaced by the number of wiggler periods $N_{w}$, the resonant frequency replaced by $\omega_{n}=2 \gamma_{z 0}^{2} n c k_{w} /\left(1+a_{w}^{2} / 2+\gamma_{z 0}^{2} \theta^{2}\right)$, and the critical frequency replaced by $\omega_{c} \simeq 3 a_{w} \gamma_{z 0}^{2} c k_{w}$, where $k_{w}$ is the wiggler wavenumber.

The radiation spectrum for a single electron scattered by a laser with $a_{0}=1$ and $N_{0}=10$ is shown in Fig. 10. The figure shows the spectral density $d^{2} I / d \hbar \omega d \Omega$ (normalized to $\alpha_{f} \gamma_{z 0}^{2}$ ) versus normalized frequency $\hat{k}=k / 2 \gamma_{z 0}^{2} k_{\beta}$ and angle $\hat{\theta}=\gamma_{z 0} \theta$, for (a) $\phi=0$ and (b) $\phi=\pi / 2$.

3) Spectrum for an Electron Bunch with Finite Energy Spread: To include the effect of energy spread, the spectral flux density is integrated over the electron energy distribution, $f(\gamma)$

$$
\frac{d^{2} I_{n T}}{d \hbar \omega d \Omega} \simeq \int d \gamma f(\gamma) \frac{d^{2} I_{n}}{d \hbar \omega d \Omega}
$$

Beam emittance is neglected since the angular width of the spectrum over the photon energies of interest is much broader than typical beam divergences.

The electron bunch produced by a LWFA will have a finite energy spread. The SM-LWFA is typically characterized by a very broad energy distribution, e.g., a Boltzmann distribution. For a standard LWFA with laser-triggered injection, the energy spread is smaller, typically a few percent. In the following, $f(\gamma)$ is assumed to be slowly varying compared to $R_{n}\left(\omega, \omega_{n}\right)$ for fixed $\omega$. For fixed $\omega$, the energy width $\Delta \gamma_{n}$ of the resonance function $R_{n}$ about the resonant energy $\gamma_{n}$ is given 

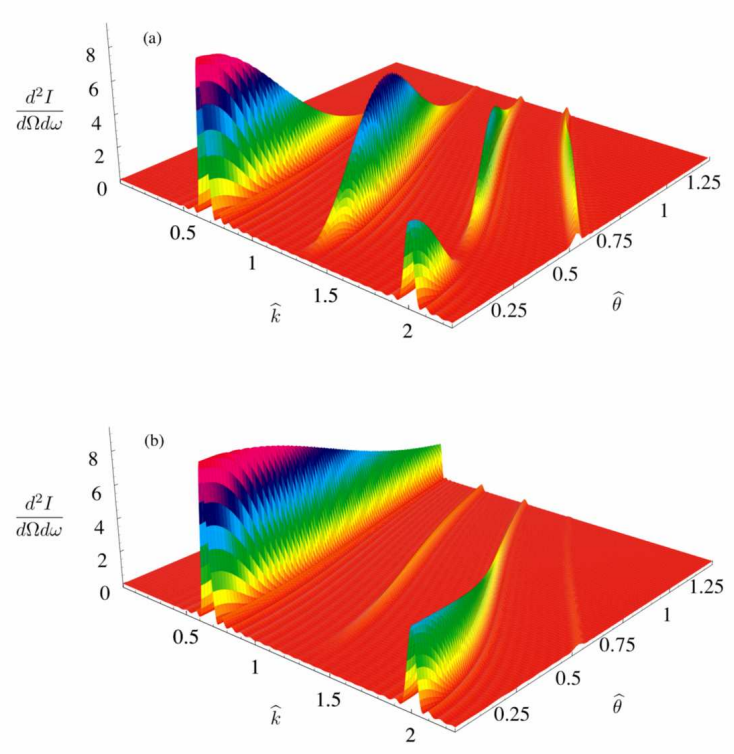

Fig. 10. Normalized spectrum $d^{2} I / d \omega \delta \Omega$ (arbitrary units) versus normalized frequency $\hat{k}$ and angle $\hat{\theta}$ from a single electron with $a_{0}=1$ and $N_{0}=10$ for (a) $\phi=0$ and (b) $\phi=\pi / 2$.

by

$$
\Delta \gamma_{n}=\int d \delta u R_{n}=\left(\frac{\gamma_{n}}{2 n N_{0}}\right)\left(1-\frac{\omega \theta^{2}}{4 n \omega_{0}}\right)^{-1}
$$

where $\delta \gamma=\gamma-\gamma_{n}$. Here, the resonant energy $\gamma_{n}$ is found by inverting the expression $\omega=\omega_{n}$, i.e.,

$$
\gamma_{n}=\left(\frac{\omega \gamma_{\perp}^{2}}{4 n \omega_{0}}\right)^{1 / 2}\left(1-\frac{\omega \theta^{2}}{4 n \omega_{0}}\right)^{-1 / 2}
$$

where $\gamma_{n}^{2} \gg \gamma_{\perp}^{2}$ and $\theta \ll 1$ have been assumed. Notice that in the backscattered direction, $\Delta \gamma_{n} / \gamma_{n}=1 / 2 n N_{0}$. Hence, the condition that $f(\gamma)$ be slowly varying compared to $R_{n}\left(\omega, \omega_{n}\right)$ for fixed $\omega$ implies that $\Delta \gamma_{n} / \gamma_{n} \ll \Delta \gamma / \gamma(\Delta \gamma / \gamma$ is the energy spread of the bunch), or $\Delta \gamma / \gamma \gg 1 / 2 n N_{0}$, which is easily satisfied for a large number of laser periods $N_{0} \gg$ $(2 n \Delta \gamma / \gamma)^{-1}$. In the limit of a large number of laser periods $(N \rightarrow \infty), R_{n} \rightarrow \Delta \gamma_{n} \delta\left(\gamma-\gamma_{n}\right)$.

The above integration over momentum can be simplified by approximating the resonance function as a delta function, i.e., $R_{n}\left(\omega, \omega_{n}\right) \simeq \Delta \gamma_{n} \delta\left(\gamma-\gamma_{n}\right)$. Hence, (37) reduces to

$$
\frac{d^{2} I_{n T}}{d \hbar \omega d \Omega} \simeq \Delta u_{n} f\left(\gamma_{n}\right) S_{n}\left(\gamma_{n}\right)
$$

where $d^{2} I_{n} / d \hbar \omega d \Omega=R_{n}\left(\omega, \omega_{n}\right) S_{n}$. The notation on the right side of the above equation indicates that in the functions $f(g)$ and $S_{n}, u$ and $\gamma$ are to be evaluated at their resonant values, $\gamma=\gamma_{n}$ and $\gamma_{z 0}=\gamma_{n}$.

The radiation spectrum for an electron beam with a $5 \mathrm{MeV}$ Boltzmann energy distribution scattering against a laser with $a_{0}=1$ and $N_{0}=10$ is shown in Fig. 11 .

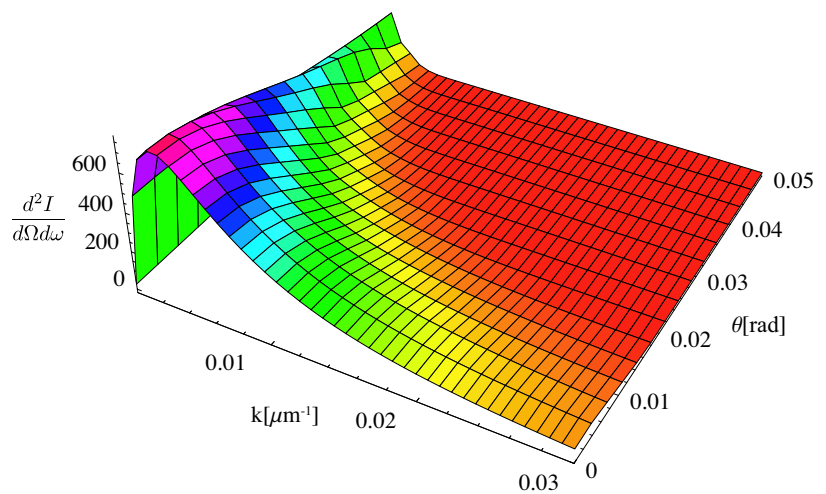

Fig. 11. Normalized spectrum $d^{2} I / d \omega \delta \Omega$ (arbitrary units) versus wavenumber $k\left[\mu \mathrm{m}^{-1}\right]$ and angle $\theta[\mathrm{rad}]$ averaged over $\phi$ with $a_{0}=1$ and $N_{0}=10$. The electron beam is assumed to have a Boltzmann energy distribution with $5 \mathrm{MeV}$ temperature.

4) Backscattered Radiation: In the backscattered direction $(\theta=0)$ in the limit $\gamma_{n} \gg \gamma_{\perp}$, the spectrum integrated over the electron energy distribution is given by [53]

$$
\begin{aligned}
\frac{d^{2} I_{n T}}{d \hbar \omega d \Omega}=2 \alpha_{f} N_{0} & \gamma_{n}^{3} f\left(\gamma_{n}\right) \frac{\alpha_{z}}{\gamma_{\perp}^{2}} \\
& \times\left[J_{(n-1) / 2}\left(\alpha_{z}\right)-J_{(n+1) / 2}\left(\alpha_{z}\right)\right]^{2}
\end{aligned}
$$

where $\gamma_{n} \simeq\left(\gamma_{\perp} / 2\right)\left(\omega / n \omega_{0}\right)^{1 / 2}$ and $\alpha_{z} \simeq n a_{0}^{2} /\left(4 \gamma_{\perp}^{2}\right)$.

5) Low Intensity Limit $\left(a_{0}^{2} \ll 1\right)$ : In the limit $a_{0}^{2} \ll 1$, only the fundamental $(n=1)$ radiation is significant. The fundamental radiation spectrum in the backscatted direction, averaged over the electron distribution, is given by

$$
\frac{d^{2} I_{T}}{d \hbar \omega d \Omega}=\alpha_{f} N_{0} \gamma_{n}^{3} f\left(\gamma_{n}\right) a_{0}^{2} / 2
$$

where $\gamma_{n} \simeq\left(\omega / 4 \omega_{0}\right)^{1 / 2}$. The total number of photons radiated over all frequencies and angles is given by

$$
N_{t o t}=(\pi / 3) \alpha_{f} N_{0} N_{b} a_{0}^{2}
$$

where $N_{b}$ is the number of electrons.

Of particular interest is the photon flux and brightness of the TS radiation. Assuming that the collection angle, $\theta_{d}$, is small $\left(\theta_{d}<(\Delta \omega / \omega)^{1 / 2} / \gamma<(1 / N)^{1 / 2} / \gamma\right)$ so that the intensity distribution is flat over the solid angle $\Delta \Omega_{d}=\pi \theta_{d}^{2}$, the number of photons intercepted in a small bandwidth, $\Delta \omega$, about $\omega$ and solid angle, $\Delta \Omega_{d}$, for an electron bunch with $N_{b}$ electrons is

$$
\begin{aligned}
N_{T} & =N_{b} \frac{d^{2} I_{T}}{d \hbar \omega d \Omega} \frac{\Delta \omega}{\omega} \pi \theta_{d}^{2} \\
& \simeq \frac{\alpha_{f}}{16} N_{b} N_{0} a_{0}^{2}\left(\frac{\omega}{\omega_{0}}\right)^{3 / 2} f\left(\gamma=\gamma_{n}\right) \frac{\Delta \omega}{\omega} \pi \theta_{d}^{2} .
\end{aligned}
$$

The average flux in photons per second, $F_{\text {ave }}$, in the collection angle $\theta_{d}$ and with bandwidth $\Delta \omega$, is $N_{T}$ multiplied by the repetition rate, $f_{r e p}$, of the laser/electron beam, i.e., $F_{\text {ave }}=N_{T} f_{\text {rep }}$. The average source brightness (in 


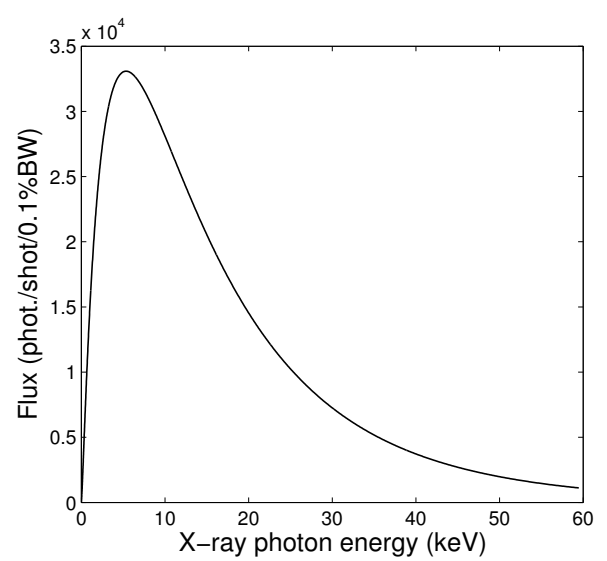

Fig. 12. Expected x-ray spectrum for the low-energy part of the electrons energy distribution (exponential distribution with $100 \%$ energy spread) within a $3 \mathrm{mrad}$ detection cone. The scattering laser is assumed to operate at 800 $\mathrm{nm}$ and to produce $1 \mathrm{~J}$ in $2 \mathrm{ps}$.

photons $/ \mathrm{s} / \mathrm{mm}^{2} / \mathrm{mrad}^{2} / 0.1 \%$ BW) is given by

$$
\begin{aligned}
B_{a v e} & =\frac{F_{a v e}}{(2 \pi)^{2} r_{b}^{2} \theta_{d}^{2}} \\
& \simeq \frac{\alpha_{f} N_{b} N_{0} a_{0}^{2}}{64 \pi r_{b}^{2}}\left(\frac{\omega}{\omega_{0}}\right)^{3 / 2} f\left(\gamma=\gamma_{n}\right) \frac{\Delta \omega}{\omega},
\end{aligned}
$$

where $r_{b}$ is the electron bunch radius. The peak flux and brightness are, respectively, $F_{p k}=F_{a v e} /\left(\tau_{x} f_{r e p}\right)$ and $B_{p k}=$ $B_{\text {ave }} /\left(\tau_{x} f_{\text {rep }}\right)$, where $\tau_{x}$ is the x-ray pulse duration, which is assumed to be approximately equal to the electron bunch duration. Note that the region of validity of (16)-(18) is for $\left(\omega / 4 \omega_{0}\right)^{1 / 2} \geq \gamma_{\min }$, where $\gamma_{\min }$ is the minimum $\gamma$ of the electrons in the distribution $f(\gamma)$.

6) Example I: Low energy $x$-ray source $(\leq 10 \mathrm{keV})$ : Laser wakefield accelerators operating in the self-modulated regime typically produce energy spectra consisting of an exponentially decreasing distribution of electrons, with a total charge of 1$10 \mathrm{nC}$ [20], [22]. A typical radiation spectrum from Thomson scattering off such an electron energy distribution is shown in Fig. 12, where the $\mathrm{x}$-ray spectrum is normalized to a detection angle $\theta_{d}=3 \mathrm{mrad}$ and a frequency bandwidth $\Delta \omega / \omega=0.1 \%$. Here, an electron energy distribution function of the form $f(\gamma) \propto \exp \left(-\gamma / \gamma_{0}\right)$ has been assumed, with $\gamma_{0}=10$ and a total charge of $5 \mathrm{nC}$, along with a $1 \mathrm{~J}, 2$ ps Ti:sapphire laser focused to a spot size of $r_{0}=12 \mu \mathrm{m}$ with $a_{0}=0.32$. The total number of scattered photons calculated from (43) for this case is $N_{\text {tot }} \simeq 1.7 \times 10^{10}$. Also shown in Table I are two other cases: a $\lambda=0.8 \mu \mathrm{m}, 1 \mathrm{~J}, 200$ ps laser with $a_{0}=0.032$; and a $\lambda=1.064 \mu \mathrm{m}, 1 \mathrm{~J}, 20 \mathrm{~ns}$ laser with $a_{0}=0.0043$.

7) Example II: High energy $x$-ray source $(\geq 100 \mathrm{keV})$ : Consider next a laser wakefield accelerator producing a narrow energy spread electron beam, such as in the proposed colliding pulse injection method [10], [12], [13], or as in the recent experiments on the channel-guided LWFA [15]. To estimate the Thomson scattered spectrum in such an LWFA, the high energy part of the electron spectrum is assumed to exhibit a narrow peak at a high energy $(50 \mathrm{MeV})$ with a total charge of $0.3 \mathrm{nC}$ and an energy spread of $3 \%$ (FWHM). The total number of scattered x-ray photons is $N_{t o t} \simeq 10^{9}$, as calculated

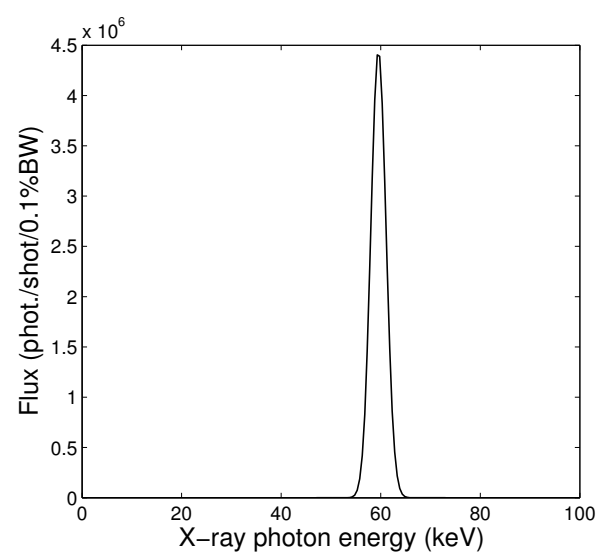

Fig. 13. Expected spectrum for the high-energy part of the electrons spectrum (narrow peak at $50 \mathrm{MeV}$ and $3 \%$ energy spread (FWHM)) within a $3 \mathrm{mrad}$ detection cone. The laser is assumed to operate at $800 \mathrm{~nm}$ and to produce 1 $\mathrm{J}$ in $2 \mathrm{ps}$.

from (43), assuming a $1 \mathrm{~J}, 2$ ps Ti:sapphire laser focused to a spot size of $r_{0}=12 \mu \mathrm{m}$ with $a_{0}=0.32$.

The spectral width of the peak radiated by a single electron is $\delta \omega / \omega=1 / N_{0}$, whereas the spread in scattered frequencies as a result of finite electron energy spread $\Delta \gamma / \gamma$ is $\delta \omega / \omega \simeq 2 \Delta \gamma / \gamma$. For a sufficiently long laser pulse with $N_{0} \gg(2 \Delta \gamma / \gamma)^{-1}$, the width of the single electron resonance function $R_{n}$ will be much narrower than the width of the electron distribution function. In this limit the scattered spectrum can be estimated using (44), and the width of the peak in the x-ray spectrum is determined by the width of the energy spread of the electrons. The expected spectrum for a detection angle of $\theta_{d}=3 \mathrm{mrad}$ and an electron beam with a narrow peak at $50 \mathrm{MeV}$ with a $3 \%$ energy spread (FWHM) is shown in Fig. 13.

Three source designs are summarized in Table I. The key difference between the three cases is the laser that is used for scattering. The first case assumes a long-pulse, Nd:YAG, Qswitched laser operating at $1064 \mathrm{~nm}$ and producing $1 \mathrm{~J}$ energy in a $20 \mathrm{~ns}$ pulse. The second and third cases use a more powerful Ti: $\mathrm{Al}_{2} \mathrm{O}_{3}$ laser operating at $800 \mathrm{~nm}$ wavelength. The laser, based on chirped pulse amplification, delivers $1 \mathrm{~J}$ energy in a pulse that is either uncompressed (case 2) or partially compressed (case 3). In all cases the interaction length is approximately the Rayleigh length $Z_{R}=\pi r_{0}^{2} / \lambda\left(N_{0} \simeq\right.$ $\left.Z_{R} / \lambda\right)$ with $r_{0}=12 \mu \mathrm{m}$, such that the total number of photons is proportional to the laser power. Furthermore, non-uniform pulse profile effects are neglected [54]. These examples show that excellent performance of a femtosecond $\mathrm{x}$-ray source with sufficient flux for various pump-probe experiments can potentially be achieved using a moderately powerful laser.

\section{CONCLUSION}

Radiation produced by laser accelerated electron bunches has been analyzed. In the long wavelength range of the electromagnetic spectrum ( $\mathrm{THz}$ radiation), the ultra-short pulse nature of the accelerated electron bunches provides the possibility of producing high energy coherent radiation. In proofof-principle experiments, coherent tranistion radiation emis- 
TABLE I

EXPECTED THOMSON SOURCE PERFORMANCE USING A 3 mrad COLLECTION ANGLE FOR THE LOW ENERGY ELECTRON DISTRIBUTION AND HIGH ENERGY ELECTRON DISTRIBUTION. THE SPECTRUM IN THE LOW ENERGY CASE IS BROAD, CENTERED AT $5 \mathrm{keV}$ AND EXTENDING FROM $1 \mathrm{keV}$ TO $40 \mathrm{keV}$. THE HIGH ENERGY CASE IS CENTERED AT $60 \mathrm{keV}$ WITH A FEW keV SPREAD.

\begin{tabular}{|c|c|c|c|c|c|c|}
\hline & \multicolumn{2}{|c|}{ YAG laser } & \multicolumn{2}{|c|}{ Uncompressed beam } & \multicolumn{2}{|c|}{ Partially compressed beam } \\
\hline Laser wavelength $[\mu m]$ & \multicolumn{2}{|c|}{1.064} & \multicolumn{2}{|c|}{0.8} & \multicolumn{2}{|c|}{0.8} \\
\hline Laser energy $[\mathrm{J}]$ & \multicolumn{2}{|c|}{1} & \multicolumn{2}{|c|}{1} & \multicolumn{2}{|c|}{1} \\
\hline Laser pulse duration & \multicolumn{2}{|c|}{$20 \mathrm{~ns}$} & \multicolumn{2}{|c|}{$200 \mathrm{ps}$} & \multicolumn{2}{|c|}{$2 \mathrm{ps}$} \\
\hline Laser intensity $a_{0}$ & \multicolumn{2}{|c|}{0.0043} & \multicolumn{2}{|c|}{0.032} & \multicolumn{2}{|c|}{0.32} \\
\hline Distribution & low energy & high energy & low energy & high energy & low energy & high energy \\
\hline Total number of x-ray photons per shot & $1.7 \times 10^{6}$ & $10^{5}$ & $1.7 \times 10^{8}$ & $10^{7}$ & $1.7 \times 10^{10}$ & $10^{9}$ \\
\hline Flux in $\theta_{d}(\mathrm{ph} / \mathrm{shot} / 0.1 \% \mathrm{BW})$ & 3.3 & 4400 & 330 & $4.4 \times 10^{5}$ & $3.3 \times 10^{4}$ & $4.4 \times 10^{6}$ \\
\hline Brightness ( $\left.\mathrm{ph} / \mathrm{shot} / \mathrm{mm}^{2} / \mathrm{mrad}^{2} / 0.1 \% \mathrm{BW}\right)$ & $10^{3}$ & $1.4 \times 10^{5}$ & $10^{5}$ & $1.4 \times 10^{7}$ & $10^{7}$ & $1.4 \times 10^{9}$ \\
\hline
\end{tabular}

sion was observed when electron bunches with a few $\mathrm{MeV}$ mean energy propagated through the plasma-vacuum boundary. Since the radiation was produced at this boundary, bunch densities, which would be reduced by space charge effects during propagation of these low density in vacuum, remained high. Due to the high amount of charge, this simple source can in principle produce 10's of $\mu \mathrm{J} /$ pulse. Diffraction effects arising from the finite transverse plasma size can significantly increase the opening angle of the radiation, limiting the amount of collected energy unless collection optics with large opening angle can be used. For laser wakefield accelerators that produce high energy electron beams (i.e., much less susceptible to space charge effects), metal foils can be used as in conventional CTR experiments and very high source efficiency can be expected.

In the short wavelength range (x-rays), two mechanisms for the generation of femtosecond $\mathrm{x}$-rays have been discussed. The first one relies on the emission of betatron radiation when a relativistic electron beam propagates inside a plasma channel in the presence of large focusing fields. The second one uses an external laser to Thomson backscatter off the relativistic electrons. For $100 \mathrm{MeV}$ class laser wakefield accelerators, betatron radiation $\mathrm{x}$-rays will be in the few $\mathrm{keV}$ range. Thomson scattered x-rays can be in the $60 \mathrm{keV}$ range using a $50 \mathrm{MeV}$ beam and a laser operating at $0.8 \mu \mathrm{m}$. Using TW-class lasers for Thomson scattering and electron beams achievable by present day laser wakefield accelerators, photon fluxes from TS (betatron radiation) on the order of $5 \times 10^{6}$ photons/shot/0.1\%BW $\left(2 \times 10^{4}\right.$ photons/shot $\left./ 0.1 \% \mathrm{BW}\right)$ are expected at $60 \mathrm{keV}(10 \mathrm{keV})$ in truly femtosecond duration pulses (10-50 fs). Such a source will have applicability for pump-probe experiments studying melting, shock propagation, and other phenomena in materials.

\section{ACKNOWLEDGMENT}

The authors would like to thank Gerry Dugan and Jerome Faure for their contributions. This work was supported by the U.S. Department of Energy under Contract No. DE-AC0376SF0098. C.G.R. Geddes acknowledges the Hertz Foundation for support.

\section{REFERENCES}

[1] E. Esarey, P. Sprangle, J. Krall, and A. Ting, "Overview of plasmabased accelerator concepts," IEEE Trans. Plasma Sci., vol. 24, no. 2, pp. 252-288, 1996.

[2] A. Modena, Z. Najmudin, A. E. Dangor, C. E. Clayton, K. A. Marsh, C. Joshi, V. Malka, C. B. Darrow, C. Danson, D. Neely, and F. N. Walsh, "Electron acceleration from the breaking of relativistic plasma waves," Nature, vol. 377, pp. 606-608, 1995.

[3] A. Ting, C. I. Moore, K. Krushelnick, C. Manka, E. Esarey, P. Sprangle, R. Hubbard, H. R. Burris, and M. Baine, "Plasma wakefield generation and electron acceleration in a self-modulated laser wakefield accelerator experiment," Phys. Plasmas, vol. 4, no. 5, pp. 1889-1899, 1997.

[4] C. Gahn, G. D. Tsakiris, A. Pukhov, J. Meyer-ter-Vehn, G. Pretzler, P. Thirolf, D. Habs, and K. J. Witte, "Multi-MeV electron beam generation by direct laser acceleration in high-density plasma channels," Phys. Rev. Lett., vol. 83, no. 23, pp. 4772-4775, December 1999.

[5] X. Wang, M. Krishnan, N. Saleh, H. Wang, and D. Umstadter, "Electron acceleration and the propagation of ultrashort high-intensity laser pulses in plasmas," Phys. Rev. Lett., vol. 84, no. 23, pp. 5324-5327, June 2000.

[6] M. I. K. Santala, Z. Najmudin, E. L. Clark, M. Tatarakis, K. Krushelnick, A. E. Dangor, V. Malka, J. Faure, R. Allott, and R. J. Clarke, "Observation of a hot high-current electron beam from a self-modulated laser wakefield accelerator," Phys. Rev. Lett., vol. 86, no. 7, pp. 1227-1230, 2001.

[7] W. P. Leemans, P. Catravas, E. Esarey, C. G. R. Geddes, C. Toth, R. Trines, C. B. Schroeder, B. A. Shadwick, J. van Tilborg, and J. Faure, "Electron-yield enhancement in a laser-wakefield accelerator driven by asymmetric laser pulses," Phys. Rev. Lett., vol. 89, p. 4802, 2002.

[8] V. Malka, S. Fritzler, E. Lefebvre, M. M. Aleonard, F. Burgy, J. P. Chambaret, J. F. Chemin, K. Krushelnick, G. Malka, S. P. D. Mangles, Z. Najmudin, M. Pittman, J. P. Rousseau, J. N. Scheurer, B. Walton, and A. E. Dangor, "Electron acceleration by a wake field forced by an intense ultrashort laser pulse," Science, vol. 298, no. 5598, pp. 15961600, 2002.

[9] D. Umstadter, J. K. Kim, and E. Dodd, "Laser injection of ultrashort electron pulses into wakefield plasma waves," Phys. Rev. Lett., vol. 76, no. 12 , pp. 2073-6, 1996.

[10] E. Esarey, R. F. Hubbard, W. P. Leemans, A. Ting, and P. Sprangle, "Electron injection into plasma wake fields by colliding laser pulses," Phys. Rev. Lett., vol. 79, no. 14, pp. 2682-2685, 1997.

[11] R. G. Hemker, K. C. Tzeng, W. B. Mori, C. E. Clayton, and T. Katsouleas, "Computer simulations of cathodeless, high-brightness electronbeam production by multiple laser beams in plasmas," Phys. Rev. E), vol. 57, no. 5, pp. 5920-8, 1998 .

[12] C. B. Schroeder, P. B. Lee, J. S. Wurtele, E. Esarey, and W. P. Leemans, "Generation of ultrashort electron bunches by colliding laser pulses," Phys. Rev. E, vol. 59, no. 5, pp. 6037-6047, 1999.

[13] G. Fubiani, E. Esarey, C. B. Schroeder, and W. P. Leemans, "Beat wave injection of electrons into plasma waves using two interfering laser pulses," Physical Review E, vol. 70, p. 016402, 2004.

[14] C. I. Moore, A. Ting, S. J. McNaught, J. Qiu, H. R. Burris, and P. Sprangle, "A laser accelerator injector based on laser ionization and ponderomotive acceleration of electrons (lipa)," Phys. Rev. Lett., vol. 82, pp. 1688-1691, 1999. 
[15] C. G. R. Geddes, C. Tóth, J. van Tilborg, E. Esarey, C. B. Schroeder, D. Bruhwiler, C. Nieter, J. Cary, and W. P. Leemans, "High quality electron beams from a plasma channel guided laser wakefield accelerator," Nature, vol. 431, pp. 538-541, 2004.

[16] S. Mangles, C. Murphy, Z. Najmudin, A. Thomas, J. Collier, A. Dangor, E. Divali, P. Foster, J. Gallacher, C. Hooker, D. Jaroszynski, A. Langley, W. Mori, P. Norreys, F. Tsung, R. Viskup, B. Walton, and K. Krushelnick, "Monoenergetic beams of relativistic electrons from intense laserplasma interactions," Nature, vol. 431, pp. 535-538, 2004.

[17] J. Faure, Y. Glinec, A. Pukhov, S. Kiselev, S. Gordienko, E. Lefebvre, J.-P. Rousseau, F. Burgy, and V. Malka, "A laser-plasma accelerator producing monoenergetic electron beams," Nature, vol. 431, pp. 541544, 2004.

[18] W. P. Leemans, D. Rodgers, P. Catravas, C. G. R. Geddes, G. Fubiani, E. Esarey, B. Shadwick, R. Donahue, and A. Smith, "Gamma-neutron activation experiments using laser wakefield accelerators," Phys. Plasmas, vol. 8, pp. 2510-2516, 2001.

[19] M. I. K. Santala, M. Zepf, F. N. Beg, E. L. Clark, A. E. Dangor, K. Krushelnick, M. Tatarakis, I. Watts, K. W. D. Ledingham, T. McCanny, I. Spencer, A. C. Machacek, R. Allott, R. Clarke, and P. A. Norreys, "Production of radioactive nuclides by energetic protons generated from intense laser-plasma interactions," Appl. Phys. Lett., vol. 78, no. 1, pp. 19-21, 2001.

[20] W. P. Leemans, C. G. R. Geddes, J. Faure, C. Tóth, J. van Tilborg, C. B. Schroeder, E. Esarey, G. Fubiani, D. Auerbach, B. Marcelis, M. A. Carnahan, R. A. Kaindl, J. Byrd, and M. Martin, "Observation of terahertz emission from a laser-plasma accelerated electron bunch crossing a plasma-vacuum boundary," Phys. Rev. Lett., vol. 91, p. 074802, 2003

[21] C. B. Schroeder, E. Esarey, J. van Tilborg, and W. P. Leemans, "Theory of coherent transition radiation generated at a plasma-vacuum interface," Phys. Rev. E, vol. 69, no. 1, p. 016501, 2004.

[22] W. P. Leemans, J. van Tilborg, J. Faure, C. G. R. Geddes, C. Tóth, C. B. Schroeder, E. Esarey, and G. Fubiani, "Terahertz radiation from laser accelerated electron bunches," Phys. Plasmas, vol. 11, pp. 2899_ 2906, 2004.

[23] E. Esarey, B. Shadwick, P. Catravas, and W. P. Leemans, "Synchrotron radiation from electron beams in plasma-focusing channels," Phys. Rev. $E$, vol. 65, p. 056505, 2002.

[24] S. Kiselev, A. Pukhov, and I. Kostyukov, "X-ray generation in strongly nonlinear plasma waves," Phys. Rev. Lett., vol. 93, no. 13, p. 135004, 2004.

[25] S. Wang, C. E. Clayton, B. E. Blue, E. S. Dodd, K. A. Marsh, W. B. Mori, C. Joshi, S. Lee, P. Muggli, T. Katsouleas, F. J. Decker, M. J. Hogan, R. H. Iverson, P. Raimondi, D. Walz, R. Siemann, and R. Assmann, "X-ray emission from betatron motion in a plasma wiggler," Phys. Rev. Lett., vol. 88, p. 135004, 2002.

[26] A. Rousse, K. T. Phuoc, R. Shah, A. Pukhov, E. Lefebvre, V. Malka, S. Kiselev, F. Burgy, J.-P. Rousseau, D. Umstadter, and D. Hulin, "Production of a kev x-ray beam from synchrotron radiation in relativistic laser-plasma interaction," Phys. Rev. Lett., vol. 93, no. 13, p. 135005, 2004.

[27] E. Esarey, S. K. Ride, and P. Sprangle, "Nonlinear thomson scattering of intense lasers from beams and plasmas," Phys. Rev. E, vol. 48, p. 3003, 1993.

[28] R. W. Schoenlein, W. P. Leemans, A. H. Chin, P. Volfbeyn, T. E. Glover, P. Balling, M. Zolotorev, K. J. Kim, S. Chattopadhyay, and C. V. Shank, "Femtosecond $\mathrm{X}$-ray pulses at 0.4 angstrom generated by 90 degrees thomson scattering: a tool for probing the structural dynamics of materials," Science, vol. 274, no. 5285, pp. 236-8, 1996.

[29] W. P. Leemans, R. W. Schoenlein, P. Volfbeyn, A. H. Chin, T. E. Glover, P. Balling, M. Zolotorev, K. J. Kim, S. Chattopadhyay, and C. V. Shank, "X-ray based subpicosecond electron bunch characterization using 90 degrees thomson scattering," Phys. Rev. Lett., vol. 77, no. 20, pp. 4182 5, 1996.

[30] — , "Interaction of relativistic electrons with ultrashort laser pulses: generation of femtosecond x-rays and microprobing of electron beams," IEEE J. Quantum Electron., vol. 33, no. 11, pp. 1925-34, 1997.

[31] I. V. Pogorelsky, I. Ben-Zvi, T. Hirose, S. Kashiwagi, V. Yakimenko, K. Kusche, P. Siddons, J. Skaritka, T. Kumita, A. Tsunemi, T. Omori, J. Urakawa, M. Washio, K. Yokoya, T. Okugi, Y. Liu, P. He, and D. Cline, "Demonstration of $8 \times 10 \mathrm{e} 18$ photons per second peaked at 1.8 angstrom in a relativistic thomson scattering experiment." Phys. Rev. ST Accel. Beams, vol. 3, p. 090702, 2000.

[32] R. P. Fischer, A. Ting, C. I. Moore, P. Sprangle, M. Baine, R. Elton, and S. Ride, "Generation of tunable, monochromatic X-rays in the laser synchrotron source experiment," in Proceedings of the 2001 Particle Accelerator Conference. Piscataway, NJ: IEEE, 2001, p. 2644

[33] W. J. Brown, S. G. Anderson, C. P. J. Barty, S. M. Betts, R. Booth, J. K. Crane, R. R. Cross, D. N. Fittinghoff, D. J. Gibson, F. V. Hartemann, E. P. Hartouni, J. Kuba, G. P. L. Sage, D. R. Slaughter, A. M. Tremaine, A. J. Wootton, P. T. Springer, and J. B. Rosenzweig, "Experimental characterization of an ultrafast thomson scattering $\mathrm{x}$-ray source with three-dimensional time and frequency-domain analysis." Phys. Rev. ST Accel. Beams, vol. 7, p. 060702, 2004.

[34] K.-J. Kim, S. Chattopadhyay, and C. Shank, "Generation of femtosecond X-rays by 90 degrees thomson scattering," Nucl. Instr. \& Meth. A, vol. 341, no. $1-3$, pp. 351-4, 1994.

[35] W. Leemans, S. Chattopadhyay, E.Esarey, A. Zholents, M. Zolotorev, A. Chin, R. Schoenlein, and C. Shank, "Femtosecond X-ray generation through relativistic electon beam-laser interaction," Comptes Rendus de l'Academie des Sciences Serie IV Physique Astrophysique, vol. 1, pp. 279-296, 2000.

[36] A. S. Weling, B. B. Hu, N. M. Froberg, and D. H. Auston, "Generation of tunable narrow-band thz radiation from large aperture photoconducting antennas," Appl. Phys. Lett., vol. 64, pp. 137-9, 1994.

[37] X.-C. Zhang, B. B. Hu, J. T. Darrow, and D. H. Auston, "Generation of femtosecond electromagnetic pulses from semiconductor surfaces." Appl. Phys. Lett., vol. 56, no. 11, pp. 1011-13, 1990.

[38] E. Budiarto, J. Margolies, S. Jeong, J. Son, and J. Bokor, "High intensity terahertz pulses at $1 \mathrm{khz}$ repetition rate," IEEE J. Quantum Electron., vol. 32, no. 10, pp. 1839-46, 1996.

[39] M. L. Ter-Mikaelian, High-energy electromagnetic processes in condensed media. New York: Wiley-Interscience, 1972.

[40] U. Happek, A. J. Sievers, and E. B. Blum, "Observation of coherent transition radiation,” Phys. Rev. Lett., vol. 67, no. 21, pp. 2962-2965, November 1991.

[41] Y. Shibata, T. Takahashi, T. Kanai, K. Ishi, M. Ikezawa, J. Ohkuma, S. Okuda, and T. Okada, "Diagnostics of an electron beam of a linear accelerator using coherent transition radiation," Phys. Rev. E, vol. 50, no. 2, pp. 1479-1484, August 1994.

[42] G. Dugan, A. Misuri, and W. Leemans, "Design and performance estimates for the l'oasis experiment magnetic spectrometers," Lawrence Berkeley National Laboratory, University of California, Berkeley, CA 94720, Tech. Rep. LBNL-49394, November 2001.

[43] C. B. Schroeder, E. Esarey, C. G. R. Geddes, C. Tóth, B. A. Shadwick, J. van Tilborg, J. Faure, and W. P. Leemans, "Frequency chirp and pulse shape effects in self-modulated laser wakefield accelerators," Phys. Plasmas, vol. 10, no. 5, pp. 2039-2046, May 2003.

[44] T. Hosokai, K. Kinoshita, A. Zhidkov, K. Nakamura, T. Watanabe, T. Ueda, H. Kotaki, M. Kando, K. Nakajima, and M. Uesaka, "Effect of a laser prepulse on a narrow-cone ejection of mev electrons from a gas jet irradiated by an ultrashort laser pulse," Phys. Rev. E, vol. 67, no. 3, p. 036407, March 2003.

[45] S.-Y. Chen, M. Krishnan, A. Maksimchuk, R. Wagner, and D. Umstadter, "Detailed dynamics of electron beams self-trapped and accelerated in a self-modulated laser wakefield," Phys. Plasmas, vol. 6, no. 12, pp. 4739-4749, December 1999.

[46] A. W. Chao, R. Pitthan, T. Tajima, and D. Yeremian, "Space charge dynamics of bright electron beams," Phys. Rev. ST Accel. Beams, vol. 6 , no. 2, p. 024201, February 2003.

[47] G. Fubiani, G. Dugan, W. Leemans, E. Esarey, and J. L. Bobin, "Semianalytical $6 \mathrm{~d}$ space charge model for dense electron bunches with large energy spreads," in Proc. of the 2002 Advanced Accelerator Concepts Workshop, C. E. Clayton and P. Muggli, Eds., vol. 647. NY: AIP, 2002, pp. 203-212.

[48] S. Fritzler, E. Lefebvre, V. Malka, F. Burgy, A. E. Dangor, K. Krushelnick, S. P. D. Mangles, Z. Najmudin, J.-P. Rousseau, and B.Walton, "Emittance measurements of a laser-wakefield-accelerated electron beam," Phys. Rev. Lett., vol. 92, p. 165006, 2004.

[49] D. Grischkowsky, S. Keiding, M. van Exter, and C. Fattinger, "Farinfrared time-domain spectroscopy with terahertz beams of dielectrics and semiconductors," J. Opt. Soc. Am. B, vol. 7, pp. 2006-2015, 1990.

[50] K. C. Tzeng, W. B. Mori, and T. Katsouleas, "Electron beam characteristics from laser-driven wave breaking," Phys. Rev. Lett., vol. 79, no. 26, pp. 5258-5261, 1997.

[51] J. D. Jackson, Classical Electrodynamics. New York: Wiley, 1975.

[52] H. Wiedemann, Particle Accelerator Phyisics. Berlin: Springer, 1995, vol. II.

[53] P. Catravas, E. Esarey, and W. P. Leemans, "Femtosecond X-rays from thomson scattering using laser wakefield accelerators," Meas. Sci. Technol., vol. 12, pp. 1828-1834, 2001. 
[54] P. A. Michel, E. Esarey, C. B. Schroeder, C. Tóth, W. P. Leemans, and D. Schneider, "Thomson scattering from laser wakefield accelerators," in Proceedings of the 2004 Advanced Accelerator Concepts Workshop, V. Yakimenko, Ed. AIP, 2004.

W.P. Leemans Dr. Wim Leemans obtained an electrical engineering/applied physics degree from the "Vrije Universiteit Brussel", Belgium in 1985, and the MS and Ph.D. degrees in electrical engineering with emphasis on plasma physics, in 1987 and 1991 respectively, from UCLA. He received the 1992 American Physical Society Simon Ramo award for outstanding doctoral thesis research work in plasma physics. In 1991 he joined the Lawrence Berkeley National Laboratory (LBNL), is Group Leader of the LOASIS Group at LBNL's Center for Beam Physics since 1994 and a senior scientist. He is responsible for two major experimental facilities: the L'OASIS lab with a multi-terawatt laser system (100 TW, $10 \mathrm{~Hz}$ being commissioned) and a radiation shielded cave, and the Beam Test Facility which houses a beam line for transporting the relativistic electron beam from the Advanced Light Source linac into a dedicated experimental area. His current research interests are in laser based advanced accelerator concepts, ultra-short x-ray pulse and terahertz generation and interaction of electron beams with plasmas. He is Chair of the ICFA Panel on Advanced and Novel Accelerators and a Fellow of the American Physical Society.

E. Esarey Dr. Eric Esarey received his B.S. degree in nuclear engineering from the University of Michigan, Ann Arbor, in 1981 and his Ph.D. degree in plasma physics from the Massachusetts Institute of Technology, Cambridge, in 1986 . He is currently a senior scientist and deputy leader of the L'OASIS program at Lawrence Berkeley National Laboratory. He has published over 100 refereed journal articles on intense laser-plasma interactions, advanced accelerators, novel radiation sources, and related subjects. He is a fellow of the American Physical Society.

J. van Tilborg Mr. Jeroen van Tilborg obtained his Master of Science Degree in Applied Physics from Eindhoven University of Technology in Eindhoven, the Netherlands in 2001. He is a candidate for the Ph.D at the same university. His thesis will consist of work performed as an experimental physisist at the L'OASIS facility of the Lawrence Berkeley National Laboratory since 2001. In 2001 he became a fellow of the Netherlands-America Foundation. Research interests include laser-plasma interaction, novel radiation sources based on relativistic particles, and laser driven particle accelerators.

P.A. Michel Dr. Pierre Michel obtained his Ph.D. from Ecole Polytechnique in Paris, France, in 2003. The thesis subject was on the coherence properties and the "plasma-induced smoothing" of an intense nanosecond laser pulse propagating through a plasma. He is currently the 2004 Lawrence Berkeley National Laboratory A.M. Sessler fellowship, and carries out experimental and theoretical research in the L'OASIS group. His research interests are laserplasma interaction, acceleration of electrons with ultraintense lasers and novel femtosecond x-ray sources.
Cs. Tóth Dr. Tóth received his M.Sc. and Ph.D. degrees in Laser Physics and Quantumelectronics from the Eötvös University, Budapest, Hungary in 1983 and 1987, respectively. Dr. Csaba Tóth started his scientific activity in the Research Institute for Solid State Physics, Budapest, Hungary, followed by numerous international collaborations in Europe (Greece, SU, and France). In 1993 and from 1995-97 he was a visiting scholar at Rice University, Houston, TX, then from1997 till 2000 he was a project scientist in the Institute for Nonlinear Science and The Wilson Group of the University of California at San Diego, CA. In November 2000 he joined the Center for Beam Physics of the Lawrence Berkeley National Laboratory (LBNL), Berkeley, California as a staff physicist, in the L'OASIS Group. Dr. Tóths research interests include the interactions of ultrashort, high intensity light pulses with matter: multiphoton processes, optical tunneling, higher-order optical harmonic generation, laserplasma interactions, acceleration of electrons and other charged particles by high peak-power laser pulses and plasma waves, development and application of new X-ray sources for imaging, time-resolved diffraction, and absorption in the pico- and femtosecond time scales. His honors include the Soros Fellowship for Young Scientists of Hungary (1990) and the Jánossy Award of the Central Research Institute for Physics, Budapest (1990). Dr. Csaba Tóth is a member of the Optical Society of America (OSA), the American and European Physical Societies (APS, EPS), the Hungarian Physical Society, and the Society for Photo-Optical Instrumentation and Engineering (SPIE).

C.B. Schroeder Dr. Carl B. Schroeder received B.S. degrees in Mathematics and Physics from the University of Maryland at College Park in 1994. He received his M.A. and Ph.D. degrees in Physics from the University of California at Berkeley in 1995 and 1999, respectively. After completing postdoctoral work at the University of California-Los Angeles and the Stanford Linear Accelerator Center, he joined the Center for Beam Physics at the Lawrence Berkeley National Laboratory (LBNL) in 2001. Dr. Schroeder is currently a staff scientist at LBNL.

C.G.R. Geddes Cameron Geddes obtained a B.A. degree in physics from Swarthmore College in 1997, and received the American Physical Society Apker award for the outstanding undergraduate thesis. From 1997 through 1999 he was a physicist at Lawrence Livermore National Laboratory. He received the M.A. degree in 2003, and is a candidate for the Ph.D. at the University of California Berkeley where he is supported by the Hertz fellowship. His thesis research is in the L'OASIS Group of Lawrence Berkeley National Laboratory, which he joined in 2000. Research interests include intense laser interactions with plasmas, laser driven advanced particle accelerators and radiation sources, and fusion.

B.A. Shadwick Dr. Brad Shadwick received his Ph.D. in Physics from the University of Texas at Austin in 1995. He is currently a scientist in the L'OASIS group at Lawrence Berkeley National Laboratory. His research interests include plasma wake field accelerators, intense laser-plasma interact ions, advanced numerical methods for fluids and plasmas, and analytical and numerical me thods for the continuous spectrum. 